\title{
A GLOBALLY CONVERGENT STABILIZED SQP METHOD
}

\author{
Philip E. Gill* $\quad$ Daniel P. Robinson ${ }^{\dagger}$
}

July 6, 2013

\begin{abstract}
Sequential quadratic programming (SQP) methods are a popular class of methods for nonlinearly constrained optimization. They are particularly effective for solving a sequence of related problems, such as those arising in mixedinteger nonlinear programming and the optimization of functions subject to differential equation constraints.

Recently, there has been considerable interest in the formulation of stabilized SQP methods, which are specifically designed to handle degenerate optimization problems. Existing stabilized SQP methods are essentially local, in the sense that both the formulation and analysis focus on the properties of the methods in a neighborhood of a solution. A new SQP method is proposed that has favorable global convergence properties yet, under suitable assumptions, is equivalent to a variant of the conventional stabilized SQP method in the neighborhood of a solution. The method combines a primal-dual generalized augmented Lagrangian function with a flexible line search to obtain a sequence of improving estimates of the solution. The method incorporates a convexification algorithm that allows the use of exact second-derivatives to define a convex quadratic programming (QP) subproblem without requiring that the Hessian of the Lagrangian be positive definite in the neighborhood of a solution. This gives the potential for fast convergence in the neighborhood of a solution. Additional benefits of the method are that each QP subproblem is regularized and the QP subproblem always has a known feasible point. Numerical experiments are presented for a subset of the problems from the CUTEr test collection.
\end{abstract}

Key words. Nonlinear programming, nonlinear constraints, augmented Lagrangian, sequential quadratic programming, SQP methods, stabilized SQP, regularized methods, primal-dual methods.

AMS subject classifications. 49J20, 49J15, 49M37, 49D37, 65F05, 65K05, 90C30

\footnotetext{
*Department of Mathematics, University of California, San Diego, La Jolla, CA 92093-0112 (pgill@ucsd.edu). Research supported in part by National Science Foundation grants DMS0915220 and DMS-1318480, and by Department of Energy grant DE-SC0002349.

${ }^{\dagger}$ Department of Applied Mathematics and Statistics, Johns Hopkins University, Baltimore, MD 21218-2682 (daniel.p.robinson@jhu.edu). Research supported in part by National Science Foundation grant DMS-1217153.
} 


\section{Introduction}

This paper is concerned with methods for the solution of optimization problems of the form

$$
\underset{x \in \mathbb{R}^{n}}{\operatorname{minimize}} f(x) \quad \text { subject to } c(x)=0, \quad x \geq 0,
$$

where $c: \mathbb{R}^{n} \mapsto \mathbb{R}^{m}$ and $f: \mathbb{R}^{n} \mapsto \mathbb{R}$ are twice-continuously differentiable. This problem formulation assumes that all general inequality constraints have been converted to equalities by the use of slack variables. Methods for solving problem (NP) are easily extended to the more general setting with $l \leq x \leq u$ (see, e.g., Section 6 ).

Some of the most efficient algorithms for nonlinear optimization are sequential quadratic programming (SQP) methods (for a survey, see, e.g., [1,24]). Conventional SQP methods find an approximate solution of a sequence of quadratic programming (QP) subproblems in which a quadratic model of the objective function is minimized subject to the linearized constraints. Convergence from any starting point is enforced by requiring the improvement in some merit function at each step. The merit function is usually a penalty or augmented Lagrangian function that defines some compromise between reducing the objective function and satisfying the constraints. SQP methods that solve the QP subproblem using an active-set method are able to capitalize on a good initial starting point, which makes them particularly effective for solving a sequence of related problems, such as those arising in the optimization of functions subject to differential equation constraints.

SQP methods have an inner/outer iteration structure, with the work for an inner iteration being dominated by the cost of solving a system of symmetric indefinite linear equations involving a subset of the variables and constraints. Some of the most successful methods use sophisticated matrix factorization updating techniques that exploit the fact that the linear equations change by only a single row and column at each inner iteration. These updating techniques are often customized for the particular QP method being used and have the benefit of providing a uniform treatment of ill-conditioning and singularity. On the negative side, it is difficult to implement conventional SQP methods so that the second derivatives of $f$ and $c$ may be used efficiently and reliably. Some of these difficulties stem from the theoretical properties of the QP subproblem, which can be nonconvex when second derivatives are used. Nonconvex QP is NP-hard - even for the calculation of a local minimizer $[11,19]$. The complexity of the QP subproblem has been a major impediment to the formulation of conventional second-derivative SQP methods (although methods based on indefinite QP have been proposed $[15,16])$. Over the years, algorithm developers have avoided this difficulty by using a convex QP defined in terms of a positive semidefinite approximate Hessian. In some cases, this QP is used to define the search direction directly $[21,22,32,45,47,48]$; in others, the QP is used to identify the constraints for an equality constrained subproblem that uses second derivatives $[29,30,41]$.

Recently, there has been considerable interest in the formulation of stabilized SQP methods, which are specifically designed to improve the convergence rate for degenerate problems $[14,31,35,43,50,51]$. Existing stabilized SQP methods are essentially 
local, in the sense that both the formulation and analysis focus on the properties of the methods in a neighborhood of a solution. In parallel with the development of stabilized SQP methods, regularized methods have been proposed that reduce the dependency on custom-built matrix factorization and updating methods for solving the QP subproblem (see, e.g., [24,39]). Regularized methods use a so-called regularization parameter to define linear equations that are always nonsingular. This feature avoids the need for specialized software to detect rank deficiency.

A seemingly different approach from tackling problem (NP) directly is to replace the constrained problem by a sequence of bound-constrained problems in which the equality constraints are included in an augmented Lagrangian objective function $[4,6,8-10,33,44]$. These methods have strong global convergence properties that require relatively weak assumptions on the problem.

In this paper we formulate and analyze a new SQP method that effectively combines the use of a bound-constrained augmented Lagrangian function with the three elements of conventional, regularized and stabilized SQP. In particular, the method has favorable global convergence properties, yet, under suitable assumptions, the method is equivalent to a conventional stabilized SQP method in the neighborhood of a solution. The method pairs the primal-dual generalized augmented Lagrangian merit function defined in [23] with a flexible line search to obtain a sequence of improving estimates of the solution. A crucial feature of the method is that the QP subproblem is based on the exact Hessian of the Lagrangian, yet has a unique bounded solution. This gives the potential for fast convergence in the neighborhood of a solution. Additional benefits of the method include: (i) each QP subproblem is regularized so that the resulting linear systems are always nonsingular and thirdparty solvers may be used; and (ii) the QP subproblem always has a known feasible point.

The paper is organized in seven sections. Section 1.2 is a review of some of the basic properties of SQP methods. In Section 2, the steps of the proposed primaldual SQP method are defined. Similarities with the conventional Hestenes-Powell augmented Lagrangian method are discussed and equivalence to stabilized SQP under certain assumptions is described. Global convergence results are established in Section 3. In Section 4, a "convexification procedure" is proposed for obtaining a QP subproblem with a bounded unique solution. In Section 5, we extend the connection established in Section 2 between the QP subproblems associated with the bound-constrained augmented Lagrangian and stabilized SQP. In particular, we show that in each case a standard active-set method generates the same sequence of QP iterates. Finally, in Section 6 some numerical experiments are presented for a simple MATLAB implementation applied to a selection of problems from the CUTEr test collection [28].

\subsection{Notation and terminology}

Unless explicitly indicated otherwise, $\|\cdot\|$ denotes the vector two-norm or its induced matrix norm. The inertia of a real symmetric matrix $A$, denoted by $\operatorname{In}(A)$, is the integer triple $\left(a_{+}, a_{-}, a_{0}\right)$ giving the number of positive, negative and zero eigen- 
values of $A$. Given vectors $a$ and $b$ with the same dimension, the vector with $i$ th component $a_{i} b_{i}$ is denoted by $a \cdot b$. Similarly, $\min (a, b)$ is a vector with components $\min \left(a_{i}, b_{i}\right)$. The vectors $e$ and $e_{j}$ denote, respectively, the column vector of ones and the $j$ th column of the identity matrix $I$. The dimensions of $e, e_{i}$ and $I$ are defined by the context. Given vectors $x$ and $y$, the long vector consisting of the elements of $x$ augmented by elements of $y$ is denoted by $(x, y)$. The $i$ th component of a vector labeled with a subscript will be denoted by $[\cdot]_{i}$, e.g., $\left[v_{F}\right]_{i}$ is the $i$ th component of the vector $v_{F}$. The subvector of components with indices in the index set $\mathcal{S}$ is denoted by $[\cdot]_{\mathcal{S}}$, e.g., $[v]_{\mathcal{S}}$ is the vector with components $v_{i}$ for $i \in \mathcal{S}$. A local solution of an optimization problem is denoted by $x^{*}$. The vector $g(x)$ is used to denote $\nabla f(x)$, the gradient of $f(x)$. The matrix $J(x)$ denotes the $m \times n$ constraint Jacobian, which has $i$ th row $\nabla c_{i}(x)^{T}$, the gradient of the $i$ th constraint function $c_{i}(x)$. The Lagrangian function associated with (NP) is $L(x, y, z)=f(x)-c(x)^{T} y-z^{T} x$, where $y$ and $z$ are $m$ - and $n$-vectors of dual variables associated with the equality constraints and bounds, respectively. The Hessian of the Lagrangian with respect to $x$ is denoted by $H(x, y)=\nabla^{2} f(x)-\sum_{i=1}^{m} y_{i} \nabla^{2} c_{i}(x)$.

\subsection{Background}

The vector-pair $\left(x^{*}, y^{*}\right)$ is called a first-order solution to problem (NP) if it satisfies

$$
c\left(x^{*}\right)=0 \text { and } \min \left(x^{*}, g\left(x^{*}\right)-J\left(x^{*}\right)^{T} y^{*}\right)=0,
$$

where $y^{*}$ are the Lagrange multipliers associated with the constraints $c(x)=0$.

Given an estimate $\left(x_{k}, y_{k}\right)$ of a primal-dual solution of (NP), a line-search SQP method computes a search direction $p_{k}=\widehat{x}_{k}-x_{k}$ such that $\widehat{x}_{k}$ is the solution (when it exists) of the quadratic program

$$
\begin{array}{ll}
\underset{x}{\operatorname{minimize}} & g_{k}^{T}\left(x-x_{k}\right)+\frac{1}{2}\left(x-x_{k}\right)^{T} \widehat{H}_{k}\left(x-x_{k}\right) \\
\text { subject to } & c_{k}+J_{k}\left(x-x_{k}\right)=0, \quad x \geq 0,
\end{array}
$$

where $c_{k}, g_{k}$ and $J_{k}$ denote the quantities $c(x), g(x)$ and $J(x)$ evaluated at $x_{k}$, and $\widehat{H}_{k}$ is some approximation of the Lagrangian Hessian $H\left(x_{k}, y_{k}\right)$. For the moment, we assume that the approximate Hessian $\widehat{H}_{k}$ is positive definite, in which case the QP subproblem (1.2) is convex. (This assumption is not required for the method proposed in Section 2.) If the Lagrange multiplier vector associated with the constraint $c_{k}+J_{k}\left(x-x_{k}\right)=0$ is given by $\widehat{y}_{k}$, then a solution $\left(\widehat{x}_{k}, \widehat{y}_{k}\right)$ of the QP subproblem (1.2) satisfies the optimality conditions

$$
c_{k}+J_{k}\left(\widehat{x}_{k}-x_{k}\right)=0 \quad \text { and } \quad \min \left(\widehat{x}_{k}, g_{k}+\widehat{H}_{k}\left(\widehat{x}_{k}-x_{k}\right)-J_{k}^{T} \widehat{y}_{k}\right)=0,
$$

which are analogous to (1.1). Given any $x \geq 0$, we define the index sets

$$
\mathcal{A}(x)=\left\{i: x_{i}=0\right\} \quad \text { and } \quad \mathcal{F}(x)=\{1,2, \ldots, n\} \backslash \mathcal{A}(x) .
$$

If $x$ is feasible for the constraints $c_{k}+J_{k}\left(x-x_{k}\right)=0$, then $\mathcal{A}(x)$ is the index set of active variables at $x$ (the active set at $x$ ) and $\mathcal{F}(x)$ is the index set of free variables at 
$x$. If the set $\mathcal{A}\left(\widehat{x}_{k}\right)$ associated with a solution of the subproblem (1.2) is known, then $\widehat{x}_{k}$ may be found by solving linear equations that represent the optimality conditions for an equality-constrained QP with the inequalities $x \geq 0$ replaced by $x_{i}=0$ for $i \in \mathcal{A}\left(\widehat{x}_{k}\right)$. In general, the optimal active set $\mathcal{A}\left(\widehat{x}_{k}\right)$ is not known in advance, and active-set QP methods generate a sequence of feasible estimates $\left(\widehat{x}^{(j)}, \widehat{y}^{(j)}\right)$ of $\left(\widehat{x}_{k}, \widehat{y}_{k}\right)$ such that $\left(\widehat{x}^{(j+1)}, \widehat{y}^{(j+1)}\right)=\left(\widehat{x}^{(j)}, \widehat{y}^{(j)}\right)+\alpha^{(j)}\left(p^{(j)}, q^{(j)}\right)$, with

$$
p_{A}^{(j)}=0 \text { and }\left(\begin{array}{cc}
\widehat{H}_{F} & J_{F}^{T} \\
J_{F} & 0
\end{array}\right)\left(\begin{array}{r}
p_{F}^{(j)} \\
-q^{(j)}
\end{array}\right)=-\left(\begin{array}{c}
{\left[g_{k}+\widehat{H}_{k}\left(\widehat{x}^{(j)}-x_{k}\right)-J_{k}^{T} \widehat{y}^{(j)}\right]_{F}} \\
c_{k}+J_{k}\left(\widehat{x}^{(j)}-x_{k}\right)
\end{array}\right) \text {, }
$$

where the quantities with subscripts " $F$ " and " $A$ " are defined in terms of the index sets $\mathcal{F}\left(\widehat{x}^{(j)}\right)$ and $\mathcal{A}\left(\widehat{x}^{(j)}\right)$, i.e., $\widehat{H}_{F}$ is the matrix of free rows and columns of $\widehat{H}_{k}, J_{F}$ is the matrix of free columns of $J_{k}$, and $p_{F}^{(j)}$ and $p_{A}^{(j)}$ are the free and fixed components of $p^{(j)}$. The step length $\alpha^{(j)}$ is chosen to ensure the feasibility of every element of $\widehat{x}^{(j+1)}$.

If the equations (1.4) are to be used to define $p^{(j)}$ and $q^{(j)}$, then it is necessary that $J_{F}$ has full rank, which is a crucial issue in the formulation of reliable methods. Two remedies are available.

- Rank-enforcing active-set methods maintain a set of indices $\mathcal{B}$ associated with a matrix of columns $J_{B}$ with rank $m$, i.e., the rows of $J_{B}$ are linearly independent. The set $\mathcal{B}$ is the complement in $\{1,2, \ldots, n\}$ of a "working set" $\mathcal{N}$ of indices that estimates the set $\mathcal{A}$ at a solution $\widehat{x}_{k}$ of (1.2). If $\mathcal{N}$ is a subset of $\mathcal{A}$, then a system analogous to (1.4) may be solved with $\mathcal{F}$ replaced by $\mathcal{B}$. The system is nonsingular because of the linear independence of the rows of $J_{B}$.

- Regularized active-set methods include a nonzero diagonal regularization term in the $(2,2)$ block of $(1.4)$. The magnitude of the regularization term is generally based on heuristic arguments.

Another important attribute associated with SQP methods is the rate of convergence to a local minimizer. If the active set at the solution $\widehat{x}_{k}$ of the QP subproblem (1.2), is the same as the active set at $x_{k}$, i.e., $\mathcal{A}\left(\widehat{x}_{k}\right)=\mathcal{A}\left(x_{k}\right)$ and $\mathcal{F}\left(\widehat{x}_{k}\right)=\mathcal{F}\left(x_{k}\right)$, then the QP solution $\left(\widehat{x}_{k}, \widehat{y}_{k}\right)$ is obtained in a single iteration. Specifically, the QP solution is given by $\left(\widehat{x}_{k}, \widehat{y}_{k}\right)=\left(x_{k}, y_{k}\right)+\left(p^{(0)}, q^{(0)}\right)$, with

$$
p_{A}^{(0)}=0 \quad \text { and } \quad\left(\begin{array}{cc}
\widehat{H}_{F} & J_{F}^{T} \\
J_{F} & 0
\end{array}\right)\left(\begin{array}{c}
p_{F}^{(0)} \\
-q^{(0)}
\end{array}\right)=-\left(\begin{array}{c}
{\left[g_{k}-J_{k}^{T} y_{k}\right]_{F}} \\
c_{k}
\end{array}\right),
$$

where $\widehat{H}_{F}, J_{F}, p_{F}^{(0)}$, and $p_{A}^{(0)}$ are as defined above, but with the index sets $\mathcal{F}\left(\widehat{x}_{k}\right)(=$ $\left.\mathcal{F}\left(x_{k}\right)\right)$ and $\mathcal{A}\left(\widehat{x}_{k}\right)\left(=\mathcal{A}\left(x_{k}\right)\right)$. The conditions (1.5) represent the Newton equations for finding a stationary point of the equality constrained problem defined in terms of the free variables. If $\widehat{H}_{k}=H\left(x_{k}, y_{k}\right)$ and $J_{F}$ has full rank in a neighborhood of a solution, then Newton's method converges at a quadratic rate. However, if $J_{F}$ does not have full rank, the equations (1.5) are singular with no unique solution. In this 
case, one remedy is to use a stabilized SQP method in which the QP subproblem (1.2) is replaced by

$$
\begin{array}{cl}
\underset{x, y}{\operatorname{minimize}} & g_{k}^{T}\left(x-x_{k}\right)+\frac{1}{2}\left(x-x_{k}\right)^{T} \widehat{H}_{k}\left(x-x_{k}\right)+\frac{1}{2} \mu_{k}\|y\|^{2} \\
\text { subject to } & c_{k}+J_{k}\left(x-x_{k}\right)+\mu_{k}\left(y-y_{k}\right)=0, \quad x \geq 0,
\end{array}
$$

where $\left\{\mu_{k}\right\}$ is a positive sequence such that $\mu_{k} \rightarrow 0$ as $x_{k} \rightarrow x^{*}$ (see, e.g., Wright [50], Hager [31], Li and Qi [38], and Fernández and Solodov [14]). The QP (1.6) is often referred to as a stabilized subproblem because of its calming effect on multiplier estimates for degenerate problems (see, e.g., [31,50]). Under certain assumptions, stabilized SQP methods exhibit fast local convergence. However, there is no guarantee of convergence to a local solution for an arbitrary starting point. Under suitable assumptions, the method proposed in this paper is guaranteed to be globally convergent and is equivalent to stabilized SQP in the limit. Result 2.1 of Section 2.1 below describes the precise relationship between the QP subproblems of the proposed method and stabilized SQP.

\section{A Regularized Primal-Dual Line-Search SQP Algorithm}

In this section, we define a regularized SQP line-search method based on the primaldual augmented Lagrangian function

$$
M^{\nu}\left(x, y ; y^{E}, \mu\right)=f(x)-c(x)^{T} y^{E}+\frac{1}{2 \mu}\|c(x)\|^{2}+\frac{\nu}{2 \mu}\left\|c(x)+\mu\left(y-y^{E}\right)\right\|^{2},
$$

where $\nu$ is a scalar, $\mu$ is the penalty parameter, and $y^{E}$ is an estimate of an optimal Lagrange multiplier vector $y^{*}$. (A trust-region-based method could also be given, but we focus on line-search methods in this paper.) The function (2.1), proposed by Robinson [46], and Gill and Robinson [23], may be derived by applying the primal-dual penalty function of Forsgren and Gill [18] to a problem in which the constraints are shifted by a constant vector (see Powell [44]). With the notation $c=c(x), g=g(x)$, and $J=J(x)$, the gradient of $M^{\nu}\left(x, y ; y^{E}, \mu\right)$ may be written as

$$
\begin{aligned}
\nabla M^{\nu}\left(x, y ; y^{E}, \mu\right) & =\left(\begin{array}{c}
g-J^{T}\left((1+\nu)\left(y^{E}-\frac{1}{\mu} c\right)-\nu y\right) \\
\nu\left(c+\mu\left(y-y^{E}\right)\right)
\end{array}\right) \\
& =\left(\begin{array}{c}
g-J^{T}(\pi+\nu(\pi-y)) \\
\nu \mu(y-\pi)
\end{array}\right)
\end{aligned}
$$

where $\pi=\pi\left(x ; y^{E}, \mu\right)$ denotes the vector-valued function

$$
\pi\left(x ; y^{E}, \mu\right)=y^{E}-\frac{1}{\mu} c(x) .
$$

Similarly, the Hessian of $M^{\nu}\left(x, y ; y^{E}, \mu\right)$ may be written as

$$
\nabla^{2} M^{\nu}\left(x, y ; y^{E}, \mu\right)=\left(\begin{array}{cc}
H(x, \pi+\nu(\pi-y))+\frac{1}{\mu}(1+\nu) J^{T} J & \nu J^{T} \\
\nu J & \nu \mu I
\end{array}\right) .
$$


Our approach is motivated by the following theorem, which shows that under certain assumptions, minimizers of problem (NP) are also minimizers of the bound constrained problem

$$
\underset{x, y}{\operatorname{minimize}} M^{\nu}\left(x, y ; y^{E}, \mu\right) \quad \text { subject to } \quad x \geq 0 .
$$

Theorem 2.1. (Robinson [46, Theorem 4.6.1]) If $\left(x^{*}, y^{*}\right)$ satisfies second-order sufficient conditions for a solution of problem (NP), then there exists a positive $\bar{\mu}$ such that, for all $0<\mu<\bar{\mu}, \nu>0$, and $y^{E}=y^{*}$, the point $\left(x^{*}, y^{*}\right)$ is a minimizer of problem (2.5).

The reader may refer to Robinson [46] and Gill and Robinson [23] for additional details. In this paper, however, we use Theorem 2.1 as motivation for the algorithm described below.

\subsection{Definition of the primal-dual search direction}

Given the $k$ th iterate $v_{k}=\left(x_{k}, y_{k}\right)$, a Lagrange multiplier estimate $y_{k}^{E}$, and a positive regularization parameter $\mu_{k}^{R}$, we first choose a symmetric matrix $\widehat{H}\left(x_{k}, y_{k}\right) \approx$ $H\left(x_{k}, y_{k}\right)$ such that $\widehat{H}\left(x_{k}, y_{k}\right)+\left(1 / \mu_{k}^{R}\right) J\left(x_{k}\right)^{T} J\left(x_{k}\right)$ is positive definite. One may choose $\widehat{H}\left(x_{k}, y_{k}\right)$ itself to be positive definite, but we explore a more sophisticated strategy in Section 4 that allows for an indefinite matrix $\widehat{H}\left(x_{k}, y_{k}\right)$ that more faithfully approximates $H\left(x_{k}, y_{k}\right)$. With this assumption on $\widehat{H}$, we may apply part (i) of Lemma 2.1 below with the quantities $H=\widehat{H}\left(x_{k}, y_{k}\right), J=J\left(x_{k}\right)$, and $\mu=\mu_{k}^{R}$, to infer that the matrix

$$
B^{\nu}\left(x_{k}, y_{k} ; \mu_{k}^{R}\right)=\left(\begin{array}{cc}
\widehat{H}\left(x_{k}, y_{k}\right)+\frac{1}{\mu_{k}^{R}}(1+\nu) J\left(x_{k}\right)^{T} J\left(x_{k}\right) & \nu J\left(x_{k}\right)^{T} \\
\nu J\left(x_{k}\right) & \nu \mu_{k}^{R} I
\end{array}\right)
$$

is a positive semidefinite approximation to the Hessian of $M^{\nu}$. Given an appropriate matrix $B^{\nu}\left(v_{k} ; \mu_{k}^{R}\right) \equiv B^{\nu}\left(x_{k}, y_{k} ; \mu_{k}^{R}\right)$, the primal-dual search direction is given by

$$
d_{k}=\widehat{v}_{k}-v_{k},
$$

where $\widehat{v}_{k}=\left(\widehat{x}_{k}, \widehat{y}_{k}\right)$ is a solution of the convex bound-constrained QP subproblem:

$$
\begin{array}{ll}
\underset{v}{\operatorname{minimize}} & \nabla M^{\nu}\left(v_{k} ; y_{k}^{E}, \mu_{k}^{R}\right)^{T}\left(v-v_{k}\right)+\frac{1}{2}\left(v-v_{k}\right)^{T} B^{\nu}\left(v_{k} ; \mu_{k}^{R}\right)\left(v-v_{k}\right) \\
\text { subject to } & v_{i} \geq 0, \quad i=1,2, \ldots, n .
\end{array}
$$

The following lemma provides the connections between the inertias of various matrices (part (i) may be used to conclude that the subproblem (2.8) is convex).

Lemma 2.1. Let $\mu, \nu$ be scalars such that $\mu>0$ and $\nu \geq 0$. Let $H$ and $J$ be matrices such that $H$ is symmetric $n \times n$ and $J$ is $m \times n$. If we define

$$
B^{\nu}=\left(\begin{array}{cc}
H+\frac{1}{\mu}(1+\nu) J^{T} J & \nu J^{T} \\
\nu J & \nu \mu I_{m}
\end{array}\right) \quad \text { and } \quad K=\left(\begin{array}{cc}
H & J^{T} \\
J & -\mu I_{m}
\end{array}\right) \text {, }
$$

then the following properties hold. 
(i) The matrix $H+\frac{1}{\mu} J^{T} J$ is positive definite if and only if

$$
\operatorname{In}\left(B^{\nu}\right)= \begin{cases}(n+m, 0,0) & \text { for } \nu>0 \\ (n, 0, m) & \text { for } \nu=0\end{cases}
$$

(ii) The matrix $H+\frac{1}{\mu} J^{T} J$ is positive definite if and only if $\operatorname{In}(K)=(n, m, 0)$.

Proof. It may be verified by direct multiplication that

$$
L^{T} B^{\nu} L=\left(\begin{array}{cc}
H+\frac{1}{\mu} J^{T} J & 0 \\
0 & \nu \mu I_{m}
\end{array}\right), \quad \text { where } \quad L=\left(\begin{array}{cc}
I_{n} & 0 \\
-\frac{1}{\mu} J & I_{m}
\end{array}\right) .
$$

The matrix $L$ is nonsingular, and Sylvester's law of inertia gives

$$
\operatorname{In}\left(B^{\nu}\right)=\operatorname{In}\left(L^{T} B^{\nu} L\right)= \begin{cases}\operatorname{In}\left(H+\frac{1}{\mu} J^{T} J\right)+(m, 0,0) & \text { for } \nu>0 \\ \operatorname{In}\left(H+\frac{1}{\mu} J^{T} J\right)+(0,0, m) & \text { for } \nu=0\end{cases}
$$

which implies the result of part (i).

To prove part (ii), consider the identity

$$
S^{T} K S=\left(\begin{array}{cc}
H+\frac{1}{\mu} J^{T} J & 0 \\
0 & -\mu I_{m}
\end{array}\right), \quad \text { where } \quad S=\left(\begin{array}{cc}
I_{n} & 0 \\
\frac{1}{\mu} J & I_{m}
\end{array}\right) .
$$

It now follows from the nonsingularity of $S$ and Sylvester's law of inertia that

$$
\operatorname{In}(K)=\operatorname{In}\left(S^{T} K S\right)=\operatorname{In}\left(H+\frac{1}{\mu} J^{T} J\right)+(0, m, 0),
$$

from which part (ii) follows directly.

A proof similar to that used for Theorem 5.1 in Section 5 may be used to show that the first-order optimality conditions for any primal-dual QP solution $\widehat{v}_{k}=$ $\left(\widehat{x}_{k}, \widehat{y}_{k}\right)$ of the bound-constrained QP (2.8) may be written in matrix form

$$
\left(\begin{array}{cc}
\widehat{H}_{F} & J_{F}^{T} \\
J_{F} & -\mu_{k}^{R} I
\end{array}\right)\left(\begin{array}{c}
{\left[\widehat{x}_{k}-x_{k}\right]_{F}} \\
-\left(\widehat{y}_{k}-y_{k}\right)
\end{array}\right)=-\left(\begin{array}{c}
{\left[g_{k}+\widehat{H}_{k} s_{k}-J_{k}^{T} y_{k}\right]_{F}} \\
c_{k}+J_{k} s_{k}+\mu_{k}^{R}\left(y_{k}-y_{k}^{E}\right)
\end{array}\right),
$$

where $c_{k}, g_{k}$ and $J_{k}$ denote the quantities $c(x), g(x)$ and $J(x)$ evaluated at $x_{k}$, and the quantities with suffix " $F$ " are defined in terms of the index set $\mathcal{F}\left(\widehat{x}_{k}\right)$, i.e., $\widehat{H}_{F}$ is the matrix of free rows and columns of $\widehat{H}_{k}=\widehat{H}\left(x_{k}, y_{k}\right)$, and $J_{F}$ is the matrix of free columns of $J_{k}$. The vector $s_{k}$ is nonpositive with components

$$
\left[s_{k}\right]_{i}=\left\{\begin{array}{cl}
-\left[x_{k}\right]_{i} & \text { if } i \in \mathcal{A}\left(\widehat{x}_{k}\right) \\
0 & \text { if } i \in \mathcal{F}\left(\widehat{x}_{k}\right)
\end{array}\right.
$$

As $\widehat{H}_{k}+\left(1 / \mu_{k}^{R}\right) J_{k}^{T} J_{k}$ is positive definite by construction, it follows immediately that the principal submatrix $\widehat{H}_{F}+\left(1 / \mu_{k}^{R}\right) J_{F}^{T} J_{F}$ is also positive definite. We may then 
apply part (ii) of Lemma 2.1 with values $H=\widehat{H}_{F}, J=J_{F}$, and $\mu=\mu_{k}^{R}$, to infer that the matrix associated with the equations (2.9) is nonsingular. It follows that if $\mathcal{A}\left(\widehat{x}_{k}\right)=\mathcal{A}\left(x_{k}\right)$, then $s_{k}$ is zero and $\left(\widehat{x}_{k}, \widehat{y}_{k}\right)$ satisfies the perturbed Newton equations

$$
\left(\begin{array}{cc}
\widehat{H}_{F} & J_{F}^{T} \\
J_{F} & -\mu_{k}^{R} I
\end{array}\right)\left(\begin{array}{c}
{\left[\widehat{x}_{k}-x_{k}\right]_{F}} \\
-\left(\widehat{y}_{k}-y_{k}\right)
\end{array}\right)=-\left(\begin{array}{c}
{\left[g_{k}-J_{k}^{T} y_{k}\right]_{F}} \\
c_{k}+\mu_{k}^{R}\left(y_{k}-y_{k}^{E}\right)
\end{array}\right) .
$$

A key property is that if $\mu_{k}^{R}=0$ and $J_{F}$ has full rank, then this equation is identical to the equation for the conventional SQP step given by (1.5). This provides the motivation to use a small penalty parameter $\mu_{k}^{R}$ for the step computation and a different larger penalty parameter $\mu_{k}$ for the merit function. In this context, $\mu_{k}^{R}$ plays the role of a regularization parameter rather than a penalty parameter, thereby providing an $O\left(\mu_{k}^{R}\right)$ estimate of the conventional SQP direction. This approach is nonstandard because a small "penalty parameter" $\mu_{k}^{R}$ is used by design, whereas conventional augmented Lagrangian-based methods attempt to keep $\mu$ as large as possible $[5,21]$.

The discussion above has established the relationship between the primal-dual bound-constrained step computation and regularized SQP. The next result formalizes the connection between the primal-dual step and the step associated with a stabilized SQP method.

Result 2.1. Let $\nu$ and $\mu_{k}^{R}$ denote fixed scalars such that $\nu \geq 0$ and $\mu_{k}^{R}>0$. Let $v_{k}=\left(x_{k}, y_{k}\right), g_{k}=g\left(x_{k}\right), c_{k}=c\left(x_{k}\right)$, and $J_{k}=J\left(x_{k}\right)$. Given a matrix $\widehat{H}_{k}=$ $\widehat{H}\left(x_{k}, y_{k}\right)$ such that $\widehat{H}_{k}+\left(1 / \mu_{k}^{R}\right) J_{k}^{T} J_{k}$ is positive definite, consider the subproblem

$$
\begin{array}{cl}
\underset{x, y}{\operatorname{minimize}} & g_{k}^{T}\left(x-x_{k}\right)+\frac{1}{2}\left(x-x_{k}\right)^{T} \widehat{H}_{k}\left(x-x_{k}\right)+\frac{1}{2} \mu_{k}^{R}\|y\|^{2} \\
\text { subject to } & c_{k}+J_{k}\left(x-x_{k}\right)+\mu_{k}^{R}\left(y-y_{k}^{E}\right)=0, \quad x \geq 0,
\end{array}
$$

which is the stabilized SQP subproblem (1.6) defined with $\mu_{k}=\mu_{k}^{R}$ and $y_{k}^{E}=y_{k}$. The following results hold.

(i) The stabilized $Q P(2.11)$ has a unique bounded primal-dual solution $\widehat{v}_{k}=$ $\left(\widehat{x}_{k}, \widehat{y}_{k}\right)$.

(ii) The unique solution $\widehat{v}_{k}=\left(\widehat{x}_{k}, \widehat{y}_{k}\right)$ of the stabilized $Q P(2.11)$ is a solution of the bound-constrained $Q P(2.8)$ for all $\nu \geq 0$. If $\nu>0$, then the stabilized solution $\widehat{v}_{k}=\left(\widehat{x}_{k}, \widehat{y}_{k}\right)$ is the unique solution of $(2.8)$.

Proof. To simplify notation, we use $\mu=\mu_{k}^{R}$. For part (i), given the particular feasible point $v_{0}=\left(x_{k}, \pi_{k}\right)$ with $\pi_{k}=y_{k}^{E}-c_{k} / \mu$, we know that every feasible point $v=(x, y)$ may be written as

$$
v=v_{0}+N w \text { for some vector } w \in \mathbb{R}^{n}, \text { where } N=\left(\begin{array}{c}
\mu I \\
-J_{k}
\end{array}\right) .
$$

The matrix $N$ is $(n+m) \times n$ with rank $n$, and its columns form a basis for the null-space of the constraint matrix $\left(\begin{array}{lll}J_{k} & \mu I\end{array}\right)$. Applying this equivalent form of $v$ to 
(2.11) gives the equivalent problem

$$
\underset{w \in \mathbb{R}^{n}}{\operatorname{minimize}} \frac{\mu}{2} w^{T}\left(\widehat{H}_{k}+\frac{1}{\mu} J_{k}^{T} J_{k}\right) w+w^{T}\left(g_{k}-J_{k}^{T} \pi_{k}\right) \text { subject to } \mu w \geq-x_{k} .
$$

The matrix $\widehat{H}_{k}+(1 / \mu) J_{k}^{T} J_{k}$ is positive definite by assumption, and it follows that the stabilized QP (2.11) is equivalent to a convex program with a strictly convex objective. The existence of a unique bounded solution follows directly.

For part (ii), we begin by stating the first-order conditions for $(x, y)$ to be a solution of the stabilized QP (2.11):

$$
\begin{aligned}
& c_{k}+J_{k}\left(x-x_{k}\right)+\mu\left(y-y_{k}^{E}\right)=0, \quad \mu y=\mu w, \\
& g_{k}+\widehat{H}_{k}\left(x-x_{k}\right)-J_{k}^{T} w-z=0, \quad z \geq 0, \\
& z \cdot x=0, \quad x \geq 0,
\end{aligned}
$$

where $w$ and $z$ denote the dual variables for the equality and inequality constraints of problem (2.11), respectively. Eliminating $w$ using the equation $w=y$ gives

$$
\begin{aligned}
& c_{k}+J_{k}\left(x-x_{k}\right)+\mu\left(y-y_{k}^{E}\right)=0, \\
& g_{k}+\widehat{H}_{k}\left(x-x_{k}\right)-J_{k}^{T} y-z=0, \quad \\
& z \cdot x=0, \quad x \geq 0, \\
& z \geq 0 .
\end{aligned}
$$

First, we prove part (ii) for the case $\nu>0$. The optimality conditions for the bound-constrained QP (2.8) are

$$
\begin{aligned}
\nabla M^{\nu}\left(v_{k} ; y_{k}^{E}, \mu\right)+B^{\nu}\left(v_{k} ; \mu\right)\left(v-v_{k}\right) & =\left(\begin{array}{l}
z \\
0
\end{array}\right), & & z \geq 0, \\
z \cdot x & =0, & & x \geq 0 .
\end{aligned}
$$

Premultiplying the equality of (2.13a) by the nonsingular matrix $T$ defined by

$$
T=\left(\begin{array}{cc}
I_{n} & -\frac{1+\nu}{\nu \mu} J_{k}^{T} \\
0 & \frac{1}{\nu} I_{m}
\end{array}\right),
$$

and using the definitions (2.2) and (2.3) yields the equivalent conditions

$$
g_{k}+\widehat{H}_{k}\left(x-x_{k}\right)-J_{k}^{T} y-z=0 \quad \text { and } \quad c_{k}+J_{k}\left(x-x_{k}\right)+\mu\left(y-y_{k}^{E}\right)=0,
$$

which are identical to the relevant equalities in (2.12). Thus, if $\nu>0$ the solutions of (2.11) and (2.8) are identical.

It remains to consider the case $\nu=0$. In this situation, the objective function of the QP (2.8) includes only the primal variables $x$, which implies that the problem may be written as

$$
\begin{aligned}
& \underset{x}{\operatorname{minimize}}\left(g_{k}-J_{k}^{T} \pi_{k}\right)^{T}\left(x-x_{k}\right)+\frac{1}{2}\left(x-x_{k}\right)^{T}\left(\widehat{H}_{k}+\frac{1}{\mu} J_{k}^{T} J_{k}\right)\left(x-x_{k}\right) \\
& \text { subject to } x \geq 0,
\end{aligned}
$$


with $y$ an arbitrary vector. Although there are infinitely many solutions of (2.8) when $\nu$ is zero, the vector $x$ associated with a particular solution $(x, y)$ is unique because it is the solution of problem (2.14) for a positive-definite matrix $\widehat{H}_{k}+\frac{1}{\mu} J_{k}^{T} J_{k}$. The optimality conditions for (2.14) are

$$
\begin{aligned}
g_{k}-J_{k}^{T} \pi_{k}+\left(\widehat{H}_{k}+\frac{1}{\mu} J_{k}^{T} J_{k}\right)\left(x-x_{k}\right) & =z, \quad z \geq 0, \\
z \cdot x & =0, \quad x \geq 0 .
\end{aligned}
$$

For the given $y_{k}$ and optimal $x$, define the $m$-vector $y$ such that

$$
y-y_{k}=-\frac{1}{\mu}\left(J_{k}\left(x-x_{k}\right)+c_{k}+\mu\left(y_{k}-y_{k}^{E}\right)\right)=-\frac{1}{\mu}\left(J_{k}\left(x-x_{k}\right)+\mu\left(y_{k}-\pi_{k}\right)\right) .
$$

Equation (2.16) and the equality of (2.15) may be combined to give the matrix equation

$$
\left(\begin{array}{c}
g_{k}-J_{k}^{T} y_{k}+2 J_{k}^{T}\left(y_{k}-\pi_{k}\right) \\
\mu\left(y_{k}-\pi_{k}\right)
\end{array}\right)+\left(\begin{array}{cc}
\widehat{H}_{k}+\frac{2}{\mu} J_{k}^{T} J_{k} & J_{k}^{T} \\
J_{k} & \mu I
\end{array}\right)\left(\begin{array}{l}
x-x_{k} \\
y-y_{k}
\end{array}\right)=\left(\begin{array}{l}
z \\
0
\end{array}\right) .
$$

Applying the nonsingular matrix $\left(\begin{array}{cc}I_{n} & -\frac{2}{\mu} J_{k}^{T} \\ 0 & I_{m}\end{array}\right)$ to both sides of this equation yields

$$
\left(\begin{array}{c}
g_{k}-J_{k}^{T} y_{k} \\
c_{k}+\mu\left(y_{k}-y_{k}^{E}\right)
\end{array}\right)+\left(\begin{array}{cc}
\widehat{H}_{k} & -J_{k}^{T} \\
J_{k} & \mu I
\end{array}\right)\left(\begin{array}{l}
x-x_{k} \\
y-y_{k}
\end{array}\right)=\left(\begin{array}{l}
z \\
0
\end{array}\right) .
$$

These equations, together with the complementarity conditions $z \cdot x=0$ of (2.15), are the optimality conditions for the stabilized QP (2.11) (cf. (2.12)). It follows that if $\nu=0$, the unique solution of (2.11) is a solution of (2.8), which is what we wanted to show.

If $\nu>0$, the uniqueness of the solution $v=(x, y)$ follows from part (i) of Lemma 2.1, which implies that the objective Hessian of the bound constrained QP (2.8) is positive definite, thereby ensuring a strictly convex QP.

\subsection{Definition of the new iterate}

Once the search direction $d_{k}=\widehat{x}_{k}-x_{k}$ has been determined, a "flexible" backtracking line search is performed on the primal-dual augmented Lagrangian. A conventional backtracking line search defines $v_{k+1}=v_{k}+\alpha_{k} d_{k}$, where $\alpha_{k}=2^{-j}$ and $j$ is the smallest nonnegative integer such that

$$
M^{\nu}\left(v_{k}+\alpha_{k} d_{k} ; y_{k}^{E}, \mu_{k}\right) \leq M^{\nu}\left(v_{k} ; y_{k}^{E}, \mu_{k}\right)+\alpha_{k} \eta_{S} d_{k}^{T} \nabla M^{\nu}\left(v_{k} ; y_{k}^{E}, \mu_{k}\right)
$$

for a given $\eta_{S} \in\left(0, \frac{1}{2}\right)$. However, this approach would suffer from the Maratos effect [40] simply because the penalty parameter $\mu_{k}$ and the regularization parameter $\mu_{k}^{R}$ used to compute the trial step have different values in general. Thus, we use a 
"flexible penalty function" based on the work of Curtis and Nocedal [12], and define $\alpha_{k}=2^{-j}$, where $j$ is the smallest nonnegative integer such that

$$
M^{\nu}\left(v_{k}+\alpha_{k} d_{k} ; y_{k}^{E}, \mu_{k}^{F}\right) \leq M^{\nu}\left(v_{k} ; y_{k}^{E}, \mu_{k}^{F}\right)+\alpha_{k} \eta_{S} \delta_{k}
$$

for some value $\mu_{k}^{F} \in\left[\mu_{k}^{R}, \mu_{k}\right]$, and $\delta_{k}$ is given by

$$
\delta_{k}=\max \left(d_{k}^{T} \nabla M^{\nu}\left(v_{k} ; y_{k}^{E}, \mu_{k}^{R}\right),-\eta_{D}\left\|d_{k}\right\|^{2}\right) \leq 0,
$$

with $\eta_{D}$ a small positive constant. The use of the second term in the definition of $\delta_{k}$ increases the chance that a step is accepted during the early iterations when $\left|d_{k}^{T} \nabla M^{\nu}\left(v_{k} ; y_{k}^{E}, \mu_{k}^{R}\right)\right|$ is large. Once an appropriate value for $\alpha_{k}$ is found, the new primal-dual solution estimate is given by

$$
x_{k+1}=x_{k}+\alpha_{k}\left(\widehat{x}_{k}-x_{k}\right) \text { and } y_{k+1}=y_{k}+\alpha_{k}\left(\widehat{y}_{k}-y_{k}\right) .
$$

In a practical algorithm, the step is reduced until the Armijo condition (2.17) is satisfied for one of the values $\mu_{k}^{F}=\mu_{k}$ or $\mu_{k}^{F}=\mu_{k}^{R}$ (where the condition for $\mu_{k}^{F}=\mu_{k}$ is tried first). The following simple argument shows that the acceptance criterion (2.17) is well-defined, i.e., the sequence $\left\{2^{-j}\right\}$ must terminate with an acceptable $\alpha_{k}$. As $v=v_{k}$ is feasible for the strictly convex problem (2.8), the search direction $d_{k}=\left(\widehat{x}_{k}-x_{k}, \widehat{y}_{k}-y_{k}\right)$ is a feasible descent direction for $M^{\nu}\left(v ; y_{k}^{E}, \mu_{k}^{R}\right)$ at $v_{k}=$ $\left(x_{k}, y_{k}\right)$. If follows from standard theory that the weakened Armijo condition (2.17) will be satisfied for $\mu_{k}^{F}=\mu_{k}^{R}$ and all $\alpha_{k}>0$ sufficiently small.

\subsection{Updating the multiplier estimate}

The QP equivalence established in Result 2.1, together with the definition of the stabilized SQP subproblem (1.6) imply that setting $y_{k}^{E}=y_{k}$ in the definition of the subproblem (2.11) (or, equivalently, in the bound-constrained QP (2.8)) makes the proposed trial step identical to that of the stabilized SQP method. This motivates an update strategy that allows the definition $y_{k}^{E}=y_{k}$ as often as possible. (The validity of this strategy is supported by the numerical results of Section 6.) The idea is to define $y_{k+1}^{E}=y_{k+1}$ for the next subproblem if the line search gives an $\left(x_{k+1}, y_{k+1}\right)$ that improves at least one of two merit functions that measure the accuracy of $\left(x_{k+1}, y_{k+1}\right)$ as an estimate of $\left(x^{*}, y^{*}\right)$. Let $\beta$ denote a small positive parameter and consider the merit functions

$$
\phi_{V}(x, y)=\eta(x)+\beta \omega(x, y), \quad \text { and } \quad \phi_{O}(x, y)=\beta \eta(x)+\omega(x, y) \text {, }
$$

where $\eta(x)$ and $\omega(x, y)$ are the feasibility violation and optimality measures

$$
\eta(x)=\|c(x)\| \quad \text { and } \quad \omega(x, y)=\left\|\min \left(x, g(x)-J(x)^{T} y\right)\right\| .
$$

These functions provide two alternative weighted measures of the accuracy of $(x, y)$ as an approximate solution of problem (NP) rather than as an approximate minimizer of $M^{\nu}$. Both measures are bounded below by zero, and are equal to zero if $v$ is a first-order solution to problem (NP). 
Given these definitions, the estimate $y_{k}^{E}$ is updated when any iterate $v_{k}=\left(x_{k}, y_{k}\right)$ satisfies either $\phi_{V}\left(v_{k}\right) \leq \frac{1}{2} \phi_{V}^{\max }$ or $\phi_{O}\left(v_{k}\right) \leq \frac{1}{2} \phi_{O}^{\max }$, where $\phi_{V}^{\max }$ and $\phi_{O}^{\max }$ are bounds that are updated throughout the solution process. To ensure global convergence, an update to $y_{k}^{E}$ forces a decrease in either $\phi_{V}^{\max }$ or $\phi_{O}^{\max }$. The idea is to choose the parameter $\beta$ of $(2.20)$ to be relatively small, say $\beta=10^{-5}$. This allows frequent updates to $y_{k}^{E}$, as shown in the numerical results of Section 6 .

Finally, $y_{k}^{E}$ is also updated if an approximate first-order solution to problem (2.5) has been found for the values $y^{E}=y_{k}^{E}$ and $\mu=\mu_{k}^{R}$. The test for optimality is

$$
\left\|\nabla_{y} M^{\nu}\left(v_{k+1} ; y_{k}^{E}, \mu_{k}^{R}\right)\right\| \leq \tau_{k} \text { and }\left\|\min \left(x_{k+1}, \nabla_{x} M^{\nu}\left(v_{k+1} ; y_{k}^{E}, \mu_{k}^{R}\right)\right)\right\| \leq \tau_{k}
$$

for some small tolerance $\tau_{k}>0$. This condition is rarely triggered in practice, but the test is needed to ensure global convergence (see Table 2 of Section 6 for statistics on the frequency of this test being satisfied). Nonetheless, if condition (2.21) is satisfied, $y_{k}^{E}$ is updated with the safeguarded estimate

$$
y_{k+1}^{E}=\max \left(-y_{\max } e, \min \left(y_{k+1}, y_{\max } e\right)\right),
$$

for some large positive scalar constant $y_{\max }$.

\subsection{Updating the penalty parameters}

As we only want to decrease $\mu_{k}^{R}$ when "close" to optimality (ignoring locally infeasible problems), we use the definition

$$
\mu_{k+1}^{R}= \begin{cases}\min \left(\frac{1}{2} \mu_{k}^{R},\left\|r_{\mathrm{opt}}\left(v_{k+1}\right)\right\|^{3 / 2}\right), & \text { if }(2.21) \text { is satisfied } \\ \min \left(\mu_{k}^{R},\left\|r_{\mathrm{opt}}\left(v_{k+1}\right)\right\|^{3 / 2}\right), & \text { otherwise }\end{cases}
$$

where $r_{\text {opt }}$ is the vector-valued function

$$
r_{\mathrm{opt}}(v)=\left(\begin{array}{c}
c(x) \\
\min \left(x, g(x)-J(x)^{T} y\right)
\end{array}\right) .
$$

The update to $\mu_{k}$ is motivated by a different goal. Namely, we wish to decrease $\mu_{k}$ only when the trial step indicates that the merit function with penalty parameter $\mu_{k}$ increases. Thus, we use the definition

$$
\mu_{k+1}= \begin{cases}\mu_{k}, & \text { if } M^{\nu}\left(v_{k+1} ; y_{k}^{E}, \mu_{k}\right) \leq M^{\nu}\left(v_{k} ; y_{k}^{E}, \mu_{k}\right)+\widehat{\alpha}_{k} \eta_{S} \delta_{k} \\ \max \left(\frac{1}{2} \mu_{k}, \mu_{k+1}^{R}\right), & \text { otherwise, }\end{cases}
$$

where $\widehat{\alpha}_{k}=\min \left(\alpha_{\min }, \alpha_{k}\right)$ for some positive $\alpha_{\min }$, and $\delta_{k}$ is defined by (2.18). The use of the scalar $\alpha_{\min }$ increases the likelihood that $\mu_{k}$ will not be decreased.

\subsection{Formal statement of the algorithm}

In this section we provide a formal statement of the proposed method as Algorithm 2.1, and include some additional details. During each iteration, the trial step 
is computed as described in Section 2.1, the solution estimate is updated as in Section 2.2, the multiplier estimate $y_{k}^{E}$ is updated as in Section 2.3, and the penalty parameters are updated as in Section 2.4. It is clear from Theorem 2.1 that it is advantageous to update the value of $y_{k}^{E}$ as often as possible in order to promote global convergence, and (from Result 2.1) to recover a stabilized SQP subproblem. To this end, there are three possibilities. First, $y_{k}^{E}$ is set to $y_{k+1}$ if $\left(x_{k+1}, y_{k+1}\right)$ is acceptable to either of the merit functions $\phi_{V}$ or $\phi_{O}$ given by (2.19). These iterates are referred to as "V-iterates" and "O-iterates", respectively. The numerical results in Section 6 show that $y_{k}^{E}$ is updated this way most of the time. Second, if $\left(x_{k+1}, y_{k+1}\right)$ is not acceptable to either of the merit functions $\phi_{V}$ or $\phi_{O}$, conditions (2.21) are used to determine if $\left(x_{k+1}, y_{k+1}\right)$ is an approximate first-order solution of the bound-constrained problem (2.5). If conditions (2.21) are satisfied, the iterate is called an "M-iterate". In this case, the regularization parameter $\mu_{k}^{R}$ and subproblem tolerance $\tau_{k}$ are decreased and $y_{k}^{E}$ is updated by $\pi\left(x_{k} ; y_{k}^{E}, \mu_{k}^{R}\right)$ as in (2.3). Finally, if neither of the first two cases occur, the multiplier estimate $y_{k}^{E}$ is fixed at its current value and the associated iterate is designated as an "F-iterate".

Algorithm 2.1. Regularized primal-dual SQP algorithm (pdSQP)

Input $\left(x_{0}, y_{0}\right)$;

Set control parameters $\alpha_{\min }>0, \eta_{S} \in(0,1), \eta_{D} \in(0,1), \tau_{\text {stop }}>0, k_{\max }>0$,

$0<\beta \ll 1, y_{\max } \gg 1$, and $\nu>0$

Initialize $y_{0}^{E}=y_{0}, \tau_{0}>0, \mu_{0}^{R}>0, \mu_{0} \in\left[\mu_{0}^{R}, \infty\right)$, and $k=0$;

Compute $f\left(x_{0}\right), c\left(x_{0}\right), g\left(x_{0}\right), J\left(x_{0}\right)$, and $H\left(x_{0}, y_{0}\right)$;

for $k=0,1,2, \ldots, k_{\max }$ do

Define $\widehat{H}\left(x_{k}, y_{k}\right) \approx H\left(x_{k}, y_{k}\right)$ such that $\widehat{H}\left(x_{k}, y_{k}\right)+\left(1 / \mu_{k}^{R}\right) J_{k}^{T} J_{k}$ is positive definite;

Solve the QP (2.11) (which is equivalent to solving (2.8)) for $\left(\widehat{x}_{k}, \widehat{y}_{k}\right)$;

Find an $\alpha_{k}$ satisfying condition (2.17) for either $\mu_{k}^{F}=\mu_{k}$ or $\mu_{k}^{F}=\mu_{k}^{R}$;

Update the primal-dual estimate $\left(x_{k+1}, y_{k+1}\right)=\left(x_{k}, y_{k}\right)+\alpha_{k}\left(\widehat{x}_{k}-x_{k}, \widehat{y}_{k}-y_{k}\right)$;

Compute $f\left(x_{k+1}\right), c\left(x_{k+1}\right), g\left(x_{k+1}\right), J\left(x_{k+1}\right)$, and $H\left(x_{k+1}, y_{k+1}\right)$;

if $\phi_{V}\left(x_{k+1}, y_{k+1}\right) \leq \frac{1}{2} \phi_{V}^{\max }$ then

$\phi_{V}^{\max }=\frac{1}{2} \phi_{V}^{\max }$

$y_{k+1}^{E}=y_{k+1} ; \quad \tau_{k+1}=\tau_{k} ;$

else if $\phi_{O}\left(x_{k+1}, y_{k+1}\right) \leq \frac{1}{2} \phi_{O}^{\max }$ then [O-iterate]

$\phi_{O}^{\max }=\frac{1}{2} \phi_{O}^{\max }$

$y_{k+1}^{E}=y_{k+1} ; \quad \tau_{k+1}=\tau_{k} ;$

else if $v_{k+1}=\left(x_{k+1}, y_{k+1}\right)$ satisfies $(2.21)$ then

$y_{k+1}^{E}=\max \left(-y_{\max } e, \min \left(y_{k+1}, y_{\max } e\right)\right) ; \quad \tau_{k+1}=\frac{1}{2} \tau_{k} ;$

else

[V-iterate]

$y_{k+1}^{E}=y_{k}^{E} ; \quad \tau_{k+1}=\tau_{k} ;$

[M-iterate]

[F-iterate $]$

end if

Update $\mu_{k+1}^{R}$ and $\mu_{k+1}$ according to (2.22) and (2.24), respectively;

if $\left\|r_{\text {opt }}\left(v_{k+1}\right)\right\| \leq \tau_{\text {stop }}$ then exit;

end (for) 


\section{Convergence}

The convergence of Algorithm 2.1 is discussed under the following assumptions.

Assumption 3.1. Each $\widehat{H}\left(x_{k}, y_{k}\right)$ is chosen so that the sequence $\left\{\widehat{H}\left(x_{k}, y_{k}\right)\right\}_{k \geq 0}$ is bounded, with $\left\{\widehat{H}\left(x_{k}, y_{k}\right)+\left(1 / \mu_{k}^{R}\right) J\left(x_{k}\right)^{T} J\left(x_{k}\right)\right\}_{k \geq 0}$ uniformly positive definite.

Assumption 3.2. The functions $f$ and $c$ are twice continuously differentiable.

Assumption 3.3. The sequence $\left\{x_{k}\right\}_{k \geq 0}$ is contained in a compact set.

In the "worst" case, i.e., when all iterates are eventually M-iterates or F-iterates, Algorithm 2.1 emulates a primal-dual augmented Lagrangian method $[7,8,46]$. Consequently, it is possible that $y_{k}^{E}$ and $\mu_{k}^{R}$ will remain fixed over a sequence of iterations, although this has been uncommon in our preliminary numerical results. The following result concerns this situation.

Theorem 3.1. Let Assumptions 3.1-3.3 hold. If there exists an integer $\widehat{k}$ such that $\mu_{k}^{R} \equiv \mu^{R}>0$ and $k$ is an $F$-iterate for all $k \geq \widehat{k}$, then the following hold for the search directions $d_{k}=\left(\widehat{x}_{k}-x_{k}, \widehat{y}_{k}-y_{k}\right)$, where $\left(\widehat{x}_{k}, \widehat{y}_{k}\right)$ is the solution of subproblem (2.8);

(i) $\left\{d_{k}\right\}_{k \geq \widehat{k}}$ are uniformly bounded;

(ii) $\left\{d_{k}\right\}_{k \geq \widehat{k}}$ are bounded away from zero; and

(iii) there exists a constant $\epsilon>0$ such that

$$
\nabla M^{\nu}\left(v_{k} ; y_{k}^{E}, \mu_{k}^{R}\right)^{T} d_{k} \leq-\epsilon \text { for all } k \geq \widehat{k}
$$

Proof. The assumptions of this theorem imply that

$$
\tau_{k} \equiv \tau>0, \quad \mu_{k}^{R}=\mu^{R}, \text { and } y_{k}^{E}=y^{E} \text { for all } k \geq \widehat{k} .
$$

First we prove part (i). As discussed in the proof of Result 2.1, it is known that the solution $\left(\widehat{x}_{k}, \widehat{y}_{k}\right)$ of $(2.8)$ satisfies

$$
d_{k}=\left(\begin{array}{c}
\widehat{x}_{k}-x_{k} \\
\widehat{y}_{k}-y_{k}
\end{array}\right)=\left(\begin{array}{c}
0 \\
\pi_{k}-y_{k}
\end{array}\right)+N_{k} w^{*}, \text { where } N_{k}=\left(\begin{array}{c}
\mu^{R} I \\
-J_{k}
\end{array}\right),
$$

and $w^{*}$ is the unique solution of

$$
\underset{w \in \mathbb{R}^{n}}{\operatorname{minimize}} \frac{1}{2} \mu^{R} w^{T}\left(\widehat{H}_{k}+\frac{1}{\mu^{R}} J_{k}^{T} J_{k}\right) w+w^{T}\left(g_{k}-J_{k}^{T} \pi_{k}\right) \quad \text { subject to } x_{k}+\mu^{R} w \geq 0,
$$

for all $k \geq \widehat{k}$. It follows from Assumption 3.1 that $\left\{d_{k}\right\}_{k \geq \widehat{k}}$ is uniformly bounded provided that the quantities $g_{k}-J_{k}^{T} \pi_{k}, N_{k}, \pi_{k}$, and $y_{k}$ are all uniformly bounded for $k \geq \widehat{k}$. The boundedness of $g_{k}-J_{k}^{T} \pi_{k}, \pi_{k}$ and $N_{k}$ follow from Assumption 3.2, Assumption 3.3, (3.1), and (2.3). It remains to prove that $\left\{y_{k}\right\}_{k \geq \widehat{k}}$ is bounded. To this end, we first note that as $\mu_{k}^{R}=\mu^{R}$ for all $k \geq \widehat{k}$, the update to $\mu_{k}$ 
given by (2.24) implies that $\mu_{k} \equiv \mu \geq \mu^{R}$ for some $\mu$ and all $k$ sufficiently large. For all subsequent iterations the primal-dual merit function is monotonically decreasing, i.e., $M^{\nu}\left(x_{k+1}, y_{k+1} ; y^{E}, \mu\right) \leq M^{\nu}\left(x_{k}, y_{k} ; y^{E}, \mu\right)$. It follows that $\left\{y_{k}\right\}_{k \geq \widehat{k}}$ must be bounded, since if there were a subsequence such that $\left\|y_{k}\right\|$ went to infinity, then for the same subsequence $M^{\nu}$ would also go to infinity because both $\left\{f_{k}-c_{k}^{T} y^{E}+\frac{1}{2 \mu}\left\|c_{k}\right\|^{2}\right\}_{k \geq \widehat{k}}$ and $\left\{c_{k}\right\}_{k \geq \widehat{k}}$ are bounded from Assumptions 3.2 and 3.3. This completes the proof of part (i).

Part (ii) is established by showing that $\left\{\left\|d_{k}\right\|\right\}_{k \geq \widehat{k}}$ is bounded away from zero. If this were not the case, there would exist a subsequence $\mathcal{S}_{1} \subseteq\{k: k \geq \widehat{k}\}$ such that $\lim _{k \in \mathcal{S}_{1}} d_{k}=0$, where $d_{k}=\left(\widehat{x}_{k}-x_{k}, \widehat{y}_{k}-y_{k}\right)$ and $\left(\widehat{x}_{k}, \widehat{y}_{k}\right)$ is a solution of problem (2.8). It follows that $d_{k}$ satisfies

$$
\left(\begin{array}{c}
\widehat{z}_{k} \\
0
\end{array}\right)=B^{\nu}\left(v_{k} ; \mu^{R}\right) d_{k}+\nabla M^{\nu}\left(v_{k} ; y^{E}, \mu^{R}\right) \quad \text { and } \quad 0=\min \left(\widehat{x}_{k}, \widehat{z}_{k}\right)
$$

for all $k \in \mathcal{S}_{1}$. We may then conclude from the definition of $B^{\nu}\left(v_{k} ; \mu^{R}\right)$ and Assumptions 3.1-3.3, and (3.1) that for $k \in \mathcal{S}_{1}$ sufficiently large, iterate $v_{k}$ will satisfy condition (2.21), be an M-iterate, and $\mu_{k}^{R}$ would be decreased. This contradicts the assumption that $\mu_{k}^{R} \equiv \mu^{R}$ for all $k \geq \widehat{k}$. It follows that $\left\{\left\|d_{k}\right\|\right\}_{k \geq \widehat{k}}$ is bounded away from zero and part (ii) holds.

The proof of part (iii) is also by contradiction. Assume that there exists a subsequence $\mathcal{S}_{2}$ of $\{k: k \geq \widehat{k}\}$ such that

$$
\lim _{k \in \mathcal{S}_{2}} \nabla M^{\nu}\left(v_{k} ; y^{E}, \mu^{R}\right)^{T} d_{k}=0
$$

where we have used (3.1) and $d_{k}$ is defined as above. As the vector $v_{k}=\left(x_{k}, y_{k}\right)$ is feasible for the convex problem $(2.8)$, and $\left(\widehat{x}_{k}, \widehat{y}_{k}\right)$ is the solution of problem (2.8) for $\nu>0$ chosen in Algorithm 2.1, it must hold that

$$
\begin{aligned}
-\nabla M^{\nu}\left(v_{k} ; y^{E}, \mu^{R}\right)^{T} d_{k} & \geq \frac{1}{2} d_{k}^{T} B^{\nu}\left(v_{k} ; \mu^{R}\right) d_{k} \\
& =\frac{1}{2} d_{k}^{T} L_{k}^{-T} L_{k}^{T} B^{\nu}\left(v_{k} ; \mu^{R}\right) L_{k} L_{k}^{-1} d_{k} \\
& =\frac{1}{2} d_{k}^{T} L_{k}^{-T}\left(\begin{array}{cc}
\widehat{H}_{k}+\frac{1}{\mu^{R}} J_{k}^{T} J_{k} & 0 \\
0 & \nu \mu^{R}
\end{array}\right) L_{k}^{-1} d_{k},
\end{aligned}
$$

where $L_{k}$ denotes the nonsingular matrix

$$
L_{k}=\left(\begin{array}{cc}
I & 0 \\
-\frac{1}{\mu^{R}} J_{k} & I
\end{array}\right), \quad \text { with } \quad L_{k}^{-1} d_{k}=\left(\begin{array}{c}
p_{k} \\
q_{k}+\frac{1}{\mu^{R}} J_{k} p_{k}
\end{array}\right),
$$

with $p_{k}=\widehat{x}_{k}-x_{k}$ and $q_{k}=\widehat{y}_{k}-y_{k}$. Assumption 3.1 yields

$$
\begin{aligned}
-\nabla M^{\nu}\left(v_{k} ; y^{E}, \mu^{R}\right)^{T} d_{k} & \geq \frac{1}{2} p_{k}^{T}\left(\widehat{H}_{k}+\frac{1}{\mu^{R}} J_{k}^{T} J_{k}\right) p_{k}+\frac{1}{2} \nu \mu^{R}\left\|q_{k}+\left(1 / \mu^{R}\right) J_{k} p_{k}\right\|^{2} \\
& \geq \lambda_{\min }\left\|p_{k}\right\|^{2}+\frac{1}{2} \nu \mu^{R}\left\|q_{k}+\left(1 / \mu^{R}\right) J_{k} p_{k}\right\|^{2},
\end{aligned}
$$


for some $\lambda_{\min }>0$. Combining this with (3.2) gives the limit

$$
\lim _{k \in \mathcal{S}_{2}} p_{k}=\lim _{k \in \mathcal{S}_{2}}\left(q_{k}+\frac{1}{\mu^{R}} J_{k} p_{k}\right)=0
$$

in which case $\lim _{k \in \mathcal{S}_{2}} q_{k}=0$ follows from Assumptions 3.2 and 3.3. This contradicts the result of part (ii) and so part (iii) must hold.

We may now state the convergence result for Algorithm 2.1.

Theorem 3.2. Let Assumptions 3.1-3.3 hold. If $v_{k}$ denotes the $k$ th iterate generated by Algorithm 2.1, then either:

(i) Algorithm 2.1 terminates with an approximate primal-dual first-order solution $v_{k}$ satisfying

$$
\left\|r_{\text {opt }}\left(v_{k}\right)\right\| \leq \tau_{\text {stop }},
$$

where $r_{\mathrm{opt}}$ is defined by (2.23); or

(ii) there exists a subsequence $\mathcal{S}$ such that $\lim _{k \in \mathcal{S}} \mu_{k}^{R}=0,\left\{y_{k}^{E}\right\}_{k \in \mathcal{S}}$ is bounded, $\lim _{k \in \mathcal{S}} \tau_{k}=0$, and for each $k \in \mathcal{S}$ the vector $v_{k+1}$ is an approximate first-order solution of (2.5) with the choice $y^{E}=y_{k}^{E}$ and $\mu=\mu_{k}^{R}$ that satisfies (2.21).

Proof. If there exists a subsequence of $\left\{\left\|r_{\text {opt }}\left(v_{k}\right)\right\|\right\}_{k \geq 0}$ that converges to zero, then clearly case (i) holds. Therefore, for the remainder of the proof, it is assumed that the sequence $\left\{\left\|r_{\text {opt }}\left(v_{k}\right)\right\|\right\}_{k \geq 0}$ is bounded away from zero.

From the definitions of a $\mathrm{V}$-iterate and O-iterate, the functions $\phi_{V}$ and $\phi_{O}$, and the update strategies for $\phi_{V}^{\max }$ and $\phi_{O}^{\max }$, we conclude that the number of $\mathrm{V}$-iterates and O-iterates must be finite. We claim that there must be an infinite number of M-iterates. To prove this, assume to the contrary that the number of M-iterates is finite, so that all iterates are F-iterates for $k$ sufficiently large. It follows from the form of the update to $\mu_{k}^{R}(2.22)$ and the assumption made in this case, that eventually $\mu_{k}^{R}$ remains constant. In this case, the update to $\mu_{k}$ given by $(2.24)$ implies that eventually, $\mu_{k}$ also remains constant. These arguments imply the existence of an integer $\widehat{k}$ such that

$$
\mu_{k}^{R} \equiv \mu^{R} \leq \mu \equiv \mu_{k}, \quad y_{k}^{E} \equiv y^{E}, \quad \tau_{k} \equiv \tau>0, \text { and } k \text { is an F-iterate for all } k \geq \widehat{k} .
$$

It follows from (2.24) that

$$
M^{\nu}\left(v_{k+1} ; y^{E}, \mu\right) \leq M^{\nu}\left(v_{k} ; y^{E}, \mu\right)+\min \left(\alpha_{\min }, \alpha_{k}\right) \eta_{S} \delta_{k} \text { for all } k \geq \widehat{k},
$$

where $\delta_{k}$ is defined by (2.18). Moreover, parts (ii) and (iii) of Theorem 3.1 ensure that $\left\{\delta_{k}\right\}_{k \geq \widehat{k}}$ is a negative sequence bounded away from zero. We also claim that $\left\{\alpha_{k}\right\}_{k \geq \widehat{k}}$ is bounded away from zero. To see this, note that parts (i) and (iii) of Theorem 3.1 and Assumption 3.2 ensure that $\left\{\alpha_{k}\right\}_{k \geq \widehat{k}}$ is bounded away from zero if a standard Armijo line search is used, i.e., if $\mu_{k}^{F}=\mu^{R}$ and $\delta_{k}=d_{k}^{T} \nabla M^{\nu}\left(v_{k} ; y^{E}, \mu^{R}\right)$ 
in (2.17). However, the computed value of $\alpha_{k}$ can be no smaller because the definition of $\delta_{k}$ is less restrictive and the use of a flexible line search makes the acceptance of a step more likely. Combining these facts with (3.3), yields

$$
M^{\nu}\left(v_{k+1} ; y^{E}, \mu\right) \leq M^{\nu}\left(v_{k} ; y^{E}, \mu\right)-\kappa \text { for all } k \geq \widehat{k} \text { and some } \kappa>0,
$$

so that

$$
\lim _{k \rightarrow \infty} M^{\nu}\left(v_{k} ; y^{E}, \mu\right)=-\infty .
$$

However, Assumptions 3.2 and 3.3 ensure that this is not possible. This contradiction implies that there must exist infinitely many M-iterations, and every iterate is an M-iterate or F-iterate for $k$ sufficiently large. Part (ii) now follows from (2.22) and the properties of the updates to $\tau_{k}$ and $y_{k}^{E}$ used for M-iterates and F-iterates in Algorithm 2.1.

The "ideal" scenario is that Algorithm 2.1 generates many V-iterates/O-iterates that converge to an approximate solution of (NP); this corresponds to part (i) of Theorem 3.2. The scenario considered in part (ii) of Theorem 3.2, i.e., the generation of infinitely many M-iterates, is the "fall-back" position for Algorithm 2.1. This result would appear to be the best that can be expected without additional assumptions, such as the satisfaction of a constraint qualification at $x^{*}$. In particular, Assumptions 3.1-3.3 do not preclude the possibility that problem (NP) is infeasible. Recent work indicates that the iterates of the stabilized SQP subproblem exhibit superlinear convergence under mild conditions (see, e.g., $[13,14,35,36]$ ). In particular, neither strict complementarity nor a constraint qualification are required. However, further analysis is required to determine the conditions under which a $\mathrm{V}$ or O-iterate is always generated in the limit, which is a necessary requirement for the proposed method to inherit the superlinear convergence rate associated with conventional stabilized SQP.

The following corollary illustrates how part (ii) of Theorem 3.2 relates to the original nonlinear problem (NP) with the assumption that the linear independence constraint qualification is satisfied (see, e.g., [42]).

Corollary 3.1. Suppose that the linear independence constraint qualification holds at all limit points generated by Algorithm 2.1. Then, Algorithm 2.1 terminates with an approximate primal-dual first-order solution $v_{k}$ satisfying

$$
\left\|r_{\text {opt }}\left(v_{k}\right)\right\| \leq \tau_{\text {stop }},
$$

where $r_{\mathrm{opt}}$ is defined by (2.23).

Proof. The result is trivially satisfied if part (i) of Theorem 3.2 holds. Thus, it may be assumed that part (ii) of Theorem 3.2 holds, which ensures the existence of a subsequence $\mathcal{S}$ of M-iterates such that $\lim _{k \in \mathcal{S}} \mu_{k}^{R}=0,\left\{y_{k}^{E}\right\}_{k \in \mathcal{S}}$ is bounded, $\lim _{k \in \mathcal{S}} \tau_{k}=0$, and for each $k \in \mathcal{S}$, the vector $v_{k+1}$ is an approximate first-order solution of (2.5) with the choice $y^{E}=y_{k}^{E}$ and $\mu=\mu_{k}^{R}$ that satisfies (2.21). Using 
Assumption 3.3 we may assume, without loss of generality, that $\lim _{k \in \mathcal{S}} x_{k}=x^{*}$ for some vector $x^{*}$. Consider the definition

$$
y^{*}=\underset{y}{\operatorname{argmin}}\left\|\left[g\left(x^{*}\right)-J\left(x^{*}\right)^{T} y\right]_{F}\right\|_{2}^{2},
$$

where the " $F$ " denotes the components corresponding to the free variables at $x^{*}$. It now follows from [46, Lemma 4.3.1] that $\left(x^{*}, y^{*}\right)$ is a first-order solution of problem (NP), and that

$$
\lim _{k \in \mathcal{S}}\left(x_{k}, y_{k}\right)=\left(x^{*}, y^{*}\right) \text {. }
$$

Moreover, it follows from Assumption 3.2 and the definition of $r_{\text {opt }}$ that (3.4) will be satisfied for $k \in \mathcal{S}$ sufficiently large.

\section{Convexification of the Bound-Constrained Subproblem}

An important aspect of the proposed method is the definition of $\widehat{H}\left(x_{k}, y_{k}\right)$, which is used to ensure that the bound constrained QP subproblem (2.8) is convex. A conventional QP subproblem defined with the Hessian of the Lagrangian is not convex, in general. To avoid solving an indefinite subproblem, most existing methods are based on solving a convex QP based on a positive-semidefinite approximation $\widehat{H}\left(x_{k}, y_{k}\right)$ of the Hessian $H\left(x_{k}, y_{k}\right)$. This convex subproblem is used to either define the search direction directly, or identify the constraints for an equality-constrained QP subproblem that uses the exact Hessian (see, e.g., [3, 21, 29]).

Here we take a different approach and define a convexified QP subproblem in terms of the exact Hessian of the Lagrangian. The convex problem is defined in such a way that if the inner iterations do not alter the active set, then the computed direction is equivalent to a second-derivative stabilized SQP direction, provided that $y_{k}^{E}=y_{k}$. The method is based on defining a symmetric matrix $\widehat{H}\left(x_{k}, y_{k}\right)$ (not necessarily positive definite) as a modification of $H\left(x_{k}, y_{k}\right)$ that gives a bounded convex primal-dual subproblem (2.8).

For the remainder of this section we focus on the solution of a single QP subproblem. Thus, to simplify notation we use $v_{k}=\left(x_{k}, y_{k}\right)=(x, y), J=J_{k}$, $H=H\left(x_{k}, y_{k}\right), \widehat{H}=\widehat{H}\left(x_{k}, y_{k}\right), B^{\nu}=B^{\nu}\left(x_{k}, y_{k} ; \mu_{k}^{R}\right)$, and $\mu=\mu_{k}^{R}$. Similarly, $J_{F}$ and $J_{A}$ denote the columns of $J$ associated with the index sets $\mathcal{F}(x)$ and $\mathcal{A}(x)$ of free and fixed variables at $x$. Throughout this section, if $M$ is a symmetric matrix, then $M_{F}$ and $M_{A}$ denote the symmetric matrices with elements $m_{i j}$ for $i, j$ in $\mathcal{F}$ and $\mathcal{A}$ respectively. The definition of $\widehat{H}$ involves certain projections defined in terms of the gradients of the bound constraints. If $P_{A}$ is the matrix whose (unit) columns are the subset of columns of the identify matrix with indices in $\mathcal{A}(x)$, then $P_{A} P_{A}^{T} x$ is the orthogonal projection of $x$ onto the bounds in $\mathcal{A}(x)$. The complementary projection may be defined in terms of the matrix $P_{F}$ with (unit) columns orthogonal to $P_{A}$. With these definitions, the matrix $P=\left(\begin{array}{ll}P_{F} & P_{A}\end{array}\right)$ defines a permutation matrix such that $J P=\left(\begin{array}{ll}J_{F} & J_{A}\end{array}\right)$.

At any given $x$, the proposed convexification gives a matrix $\widehat{H}$ of the form

$$
\widehat{H}=H+E+D,
$$


where $E$ is a symmetric positive semidefinite matrix, and $D$ is a positive-semidefinite diagonal. We emphasize that $\widehat{H}$ itself is not necessarily positive definite.

First, we consider the definition of $E$. Let $K$ and $K_{F}$ denote the matrices

$$
K=\left(\begin{array}{cc}
H+E & J^{T} \\
J & -\mu I
\end{array}\right) \quad \text { and } \quad K_{F}=\left(\begin{array}{cc}
H_{F}+E_{F} & J_{F}^{T} \\
J_{F} & -\mu I
\end{array}\right) .
$$

We are particularly interested in matrices $E$ that endow $K_{F}$ with the property of second-order consistency.

Definition 4.1. If $G$ is a symmetric matrix of order $r$ and $C$ is $m \times r$, then the matrix $\left(\begin{array}{cc}G & C^{T} \\ C & -\mu I_{m}\end{array}\right)$ is said to be second-order consistent if it has inertia $(r, m, 0)$.

The idea is to define $E_{F}$ so that the matrix $K_{F}$ of (4.2) is second-order consistent. Once $E_{F}$ has been defined, the full matrix $E$ is given by

$$
E=P_{F} E_{F} P_{F}^{T}
$$

A suitable modification $E_{F}$ may be based on some variant of the symmetric indefinite factorization of the matrix

$$
\left(\begin{array}{cc}
H_{F} & J_{F}^{T} \\
J_{F} & -\mu I
\end{array}\right) .
$$

Appropriate methods include: (i) the inertia controlling $\mathrm{LBL}^{T}$ factorization (Forsgren [17], Forsgren and Gill [18]); (ii) an $\mathrm{LBL}^{T}$ factorization with pivot modification (Gould [27]); (iii) tile preordering in conjunction with pivot modification (Gill and Wong [25]); and (iv) a conventional $\mathrm{LBL}^{T}$ factorization of $H_{F}+\sigma I_{F}$ for some nonnegative scalar $\sigma$ (Wächter and Biegler [49]). In each case, the modification $E$ is zero if the matrix (4.4) is second-order consistent.

The following lemma establishes the main property of the matrix $H+E$.

Lemma 4.1. Given the matrix $K_{F}$ of (4.2), let $\bar{H}_{F}$ denote the symmetric matrix $H_{F}+E_{F}$ with $E_{F}$ positive semidefinite. If $K_{F}$ is second-order consistent, then the matrix

$$
\left(\begin{array}{cc}
\bar{H}_{F}+\frac{1}{\mu}(1+\nu) J_{F}^{T} J_{F} & \nu J_{F}^{T} \\
\nu J_{F} & \nu \mu I
\end{array}\right)
$$

is positive definite for $\nu>0$ and positive semidefinite for $\nu=0$.

Proof. Since $K_{F}$ is second-order consistent, it follows from part (ii) of Lemma 2.1 with the choice $H=\bar{H}_{F}$ and $J=J_{F}$ that $\bar{H}_{F}+(1 / \mu) J_{F}^{T} J_{F}$ is positive definite. The result now follows from part (i) of Lemma 2.1.

The second modification $D$ is a positive-semidefinite diagonal matrix defined in terms of the gradients of the constraints with indices in $\mathcal{A}(x)$. In particular,

$$
D=\frac{1}{\mu_{A}} P_{A} P_{A}^{T}
$$

where $\mu_{A}$ is a small positive scalar. The properties of the matrix $\widehat{H}=H+E+D$ are established in Theorem 4.1 below, which requires two auxiliary results. 
Lemma 4.2. Let $K_{A}$ denote the matrix

$$
K_{A}=\left(\begin{array}{ccc}
\bar{H}+\frac{1}{\mu}(1+\nu) J^{T} J & \nu J^{T} & P_{A} \\
\nu J & \nu \mu I & 0 \\
P_{A}^{T} & 0 & 0
\end{array}\right) \text {, where } \bar{H}=H+E,
$$

and the $n_{A}$ rows of $P_{A}^{T}$ comprise the gradients of the bounds in $\mathcal{A}(x)$. Then

$$
\operatorname{In}\left(K_{A}\right)=\left(n_{A}, n_{A}, 0\right)+\operatorname{In}\left(\begin{array}{cc}
\bar{H}_{F}+\frac{1}{\mu}(1+\nu) J_{F}^{T} J_{F} & \nu J_{F}^{T} \\
\nu J_{F} & \nu \mu I
\end{array}\right) .
$$

Proof. Applying the column permutation $P=\left(\begin{array}{ll}P_{F} & P_{A}\end{array}\right)$ yields

$$
\left(\begin{array}{ccc}
P^{T} & 0 & 0 \\
0 & I_{m} & 0 \\
0 & 0 & I_{A}
\end{array}\right) K_{A}\left(\begin{array}{ccc}
P & 0 & 0 \\
0 & I_{m} & 0 \\
0 & 0 & I_{A}
\end{array}\right)=\left(\begin{array}{cccc}
\widetilde{H}_{F} & \widetilde{H}_{O} & \nu J_{F}^{T} & 0 \\
\widetilde{H}_{O} & \widetilde{H}_{A} & \nu J_{A}^{T} & I_{A} \\
\nu J_{F} & \nu J_{A} & \nu \mu I & 0 \\
0 & I_{A} & 0 & 0
\end{array}\right)
$$

where $\widetilde{H}_{F}, \widetilde{H}_{A}$, and $\widetilde{H}_{O}$ are the diagonal and off-diagonal blocks of the partition

$$
\widetilde{H} \equiv P^{T}\left(\bar{H}+\frac{1}{\mu}(1+\nu) J^{T} J\right) P=\left(\begin{array}{cc}
\widetilde{H}_{F} & \widetilde{H}_{O} \\
\widetilde{H}_{O}^{T} & \widetilde{H}_{A}
\end{array}\right) .
$$

Note that $\widetilde{H}_{F}=\bar{H}_{F}+\frac{1}{\mu}(1+\nu) J_{F}^{T} J_{F}$. The matrix of (4.7) is similar (via symmetric permutations) to

$$
\left(\begin{array}{cccc}
0 & I_{A} & 0 & 0 \\
I_{A} & \widetilde{H}_{A} & \widetilde{H}_{O}^{T} & \nu J_{A}^{T} \\
0 & \widetilde{H}_{O} & \widetilde{H}_{F} & \nu J_{F}^{T} \\
0 & \nu J_{A} & \nu J_{F} & \nu \mu I
\end{array}\right)=L\left(\begin{array}{cccc}
0 & I_{A} & 0 & 0 \\
I_{A} & \widetilde{H}_{A} & 0 & 0 \\
0 & 0 & \widetilde{H}_{F} & \nu J_{F}^{T} \\
0 & 0 & \nu J_{F} & \nu \mu I
\end{array}\right) L^{T},
$$

where $L$ is the nonsingular matrix

$$
L=\left(\begin{array}{cccc}
I_{A} & 0 & 0 & 0 \\
0 & I_{A} & 0 & 0 \\
\widetilde{H}_{O} & 0 & I_{F} & 0 \\
\nu J_{A} & 0 & 0 & I
\end{array}\right)
$$

Sylvester's law of inertia gives

$$
\begin{aligned}
\operatorname{In}\left(K_{A}\right) & =\operatorname{In}\left(\begin{array}{cc}
0 & I_{A} \\
I_{A} & \widetilde{H}_{A}
\end{array}\right)+\operatorname{In}\left(\begin{array}{cc}
\widetilde{H}_{F} & \nu J_{F}^{T} \\
\nu J_{F} & \nu \mu I
\end{array}\right) \\
& =\left(n_{A}, n_{A}, 0\right)+\operatorname{In}\left(\begin{array}{cc}
\bar{H}_{F}+\frac{1}{\mu}(1+\nu) J_{F}^{T} J_{F} & \nu J_{F}^{T} \\
\nu J_{F} & \nu \mu I
\end{array}\right),
\end{aligned}
$$

as required.

For a proof of the following lemma, see, e.g., Gill and Robinson [23, Theorem 3.1]. 
Lemma 4.3. Let $G$ be a symmetric matrix of order $r$. Let $C$ be an $m \times r$ matrix with rank $m$. If the matrix $\left(\begin{array}{cc}G & C^{T} \\ C & 0\end{array}\right)$ has inertia $(r, m, 0)$, then $G+\frac{1}{\mu} C^{T} C$ is positive definite for all $\mu>0$ sufficiently small.

Theorem 4.1. If the KKT matrix $K_{F}(4.2)$ is second-order consistent, then the matrix

$$
B^{\nu}=\left(\begin{array}{cc}
\widehat{H}+\frac{1}{\mu}(1+\nu) J^{T} J & \nu J^{T} \\
\nu J & \nu \mu I
\end{array}\right), \quad \text { with } \quad \widehat{H}=H+E+D, \text { and } \nu>0
$$

is positive definite for all sufficiently small positive $\mu_{A}$, where $D$ is defined by (4.6).

Proof. Consider the matrix

$$
K_{A}=\left(\begin{array}{ccc}
\bar{H}+\frac{1}{\mu}(1+\nu) J^{T} J & \nu J^{T} & P_{A} \\
\nu J & \nu \mu I & 0 \\
P_{A}^{T} & 0 & 0
\end{array}\right), \text { where } \bar{H}=H+E,
$$

and $P_{A}$ contains the unit vectors associated with the active bounds. Then, it follows from Lemma 4.2 and Lemma 4.1 that

$$
\begin{aligned}
\operatorname{In}\left(K_{A}\right) & =\left(n_{A}, n_{A}, 0\right)+\operatorname{In}\left(\begin{array}{cc}
\bar{H}_{F}+\frac{1}{\mu}(1+\nu) J_{F}^{T} J_{F} & \nu J_{F}^{T} \\
\nu J_{F} & \nu \mu I
\end{array}\right) \\
& =\left(n_{A}, n_{A}, 0\right)+\left(n_{F}+m, 0,0\right) \\
& =\left(n+m, n_{A}, 0\right) .
\end{aligned}
$$

This identity implies that $K_{A}$ satisfies the conditions of Lemma 4.3 with

$$
G=\left(\begin{array}{cc}
\bar{H}+\frac{1}{\mu}(1+\nu) J^{T} J & \nu J^{T} \\
\nu J & \nu \mu I
\end{array}\right) \quad \text { and } \quad C^{T}=\left(\begin{array}{c}
P_{A} \\
0
\end{array}\right)
$$

in which case, the matrix

$$
G+\frac{1}{\mu_{A}} C^{T} C=\left(\begin{array}{cc}
\bar{H}+\frac{1}{\mu}(1+\nu) J^{T} J & \nu J^{T} \\
\nu J & \nu \mu I
\end{array}\right)+\frac{1}{\mu_{A}}\left(\begin{array}{c}
P_{A} \\
0
\end{array}\right)\left(\begin{array}{ll}
P_{A}^{T} & 0
\end{array}\right)=B^{\nu}
$$

is positive definite for $\mu_{A}>0$ sufficiently small, which completes the proof.

Theorem 4.1 implies that

$$
B^{\nu}=\left(\begin{array}{cc}
\widehat{H}+\frac{1}{\mu}(1+\nu) J^{T} J & \nu J^{T} \\
\nu J & \nu \mu I
\end{array}\right) \quad \text { with } \quad \widehat{H}=H+E+D,
$$

is an appropriate positive-definite Hessian for the convexified QP. The final result of this section shows that if the final QP active set is the same as the active set at $x_{k}$, and the perturbed KKT matrix defined with the Hessian $H\left(x_{k}, y_{k}\right)$ and Jacobian $J\left(x_{k}\right)$ is second-order consistent (which will hold near a solution satisfying the strong second-order conditions for optimality), then the QP step is the solution of the QP subproblem defined in terms of $H\left(x_{k}, y_{k}\right)$ and $J\left(x_{k}\right)$. 
Theorem 4.2. Let $\widehat{v}_{k}=\left(\widehat{x}_{k}, \widehat{y}_{k}\right)$ denote the unique solution of the QP subproblem (2.8) defined at $v_{k}=\left(x_{k}, y_{k}\right)$. Let $H_{F}$ denote the matrix of rows and columns of $H\left(x_{k}, y_{k}\right)$ corresponding to the set of free variables $\mathcal{F}\left(x_{k}\right)$. Similarly, let $J_{F}$ denote the matrix of free columns of $J\left(x_{k}\right)$. If the matrix

$$
\left(\begin{array}{cc}
H_{F} & J_{F}^{T} \\
J_{F} & -\mu_{k}^{R} I
\end{array}\right)
$$

is second-order consistent, and $\mathcal{A}\left(\widehat{x}_{k}\right)=\mathcal{A}\left(x_{k}\right)$, then $\left(\widehat{x}_{k}, \widehat{y}_{k}\right)$ satisfies the perturbed Newton equations

$$
\left(\begin{array}{cc}
H_{F} & J_{F}^{T} \\
J_{F} & -\mu_{k}^{R} I
\end{array}\right)\left(\begin{array}{c}
{\left[\widehat{x}_{k}-x_{k}\right]_{F}} \\
-\left(\widehat{y}_{k}-y_{k}\right)
\end{array}\right)=-\left(\begin{array}{c}
{\left[g\left(x_{k}\right)-J\left(x_{k}\right)^{T} y_{k}\right]_{F}} \\
c\left(x_{k}\right)+\mu_{k}^{R}\left(y_{k}-y_{k}^{E}\right)
\end{array}\right) .
$$

Proof. As $\mathcal{A}\left(\widehat{x}_{k}\right)=\mathcal{A}\left(x_{k}\right)$, it follows from (2.10) that the QP solution $\left(\widehat{x}_{k}, \widehat{y}_{k}\right)$ satisfies the equations

$$
\left(\begin{array}{cc}
\widehat{H}_{F} & J_{F}^{T} \\
J_{F} & -\mu_{k}^{R} I
\end{array}\right)\left(\begin{array}{c}
{\left[\widehat{x}_{k}-x_{k}\right]_{F}} \\
-\left(\widehat{y}_{k}-y_{k}\right)
\end{array}\right)=-\left(\begin{array}{c}
{\left[g\left(x_{k}\right)-J\left(x_{k}\right)^{T} y_{k}\right]_{F}} \\
c\left(x_{k}\right)+\mu_{k}^{R}\left(y_{k}-y_{k}^{E}\right)
\end{array}\right) .
$$

If $E_{k}$ and $D_{k}$ denote the modifications (4.3) and (4.6) associated with $H\left(x_{k}, y_{k}\right)$, then

$$
\widehat{H}_{F}=P_{F}^{T} \widehat{H}\left(x_{k}, y_{k}\right) P_{F}=P_{F}^{T}\left(H\left(x_{k}, y_{k}\right)+E_{k}+D_{k}\right) P_{F}=H_{F}+E_{F} .
$$

As the perturbed Newton KKT matrix (4.8) is second-order consistent it holds that $E_{F}=0$ and $\widehat{H}_{F}=H_{F}$, as required.

\section{Equivalence of the iterates of an active-set method}

In this section we consider additional connections between the bound constrained QP subproblem (2.8) and the stabilized SQP subproblem (2.11). Result 2.1 implies that if $\nu>0$ then the solutions of subproblems (2.8) and (2.11) are unique and identical. Under the conditions of Result 2.1, $\widehat{x}_{k}$ is a unique solution of (2.8) when $\nu=0$, even though the solution pair $\left(\widehat{x}_{k}, \widehat{y}_{k}\right)$ is not unique. In this case, there is a particular solution pair that is identical to the unique solution of (2.11). This analysis is extended below to establish the relationship between the iterates when an active-set method is applied to each problem.

In all that follows, the indices associated with the SQP iteration are omitted and it will be assumed that the constraints of the QP involve the constraints linearized at the point $\bar{x}$. In all cases, the suffix $j$ will be reserved for the iteration index of the QP algorithm. 


\subsection{An active-set method}

We start by defining a "conventional" active-set method on a generic convex QP with constraints written in standard form. The problem format is

$$
\begin{aligned}
\underset{x}{\operatorname{minimize}} & \mathcal{Q}(x)=g^{T}(x-\bar{x})+\frac{1}{2}(x-\bar{x})^{T} H(x-\bar{x}) \\
\text { subject to } & c+A(x-\bar{x})=0, \quad x \geq 0
\end{aligned}
$$

where $\bar{x}, c, A, g$ and $H$ are constant. Throughout, we assume that the constraints are feasible, i.e., there exists at least one nonnegative $x$ such that $c+A(x-\bar{x})=0$.

Given a feasible $x_{0}$, active-set methods generate a feasible sequence $\left\{x_{j}\right\}$ such that $\mathcal{Q}\left(x_{j+1}\right) \leq \mathcal{Q}\left(x_{j}\right)$ with $x_{j+1}=x_{j}+\alpha_{j} p_{j}$. Let the index sets $\mathcal{A}$ and $\mathcal{F}$ be defined as in (1.3). At the start of the $j$ th QP iteration, given primal-dual iterates $\left(x_{j}, w_{j}\right)$, new estimates $\left(x_{j}+p_{j}, w_{j}+q_{j}\right)$ are defined by solving a QP formed by fixing the variables with indices in $\mathcal{A}\left(x_{j}\right)$ and defining $p_{j}$ such that $x_{j}+p_{j}$ minimizes $\mathcal{Q}(x)$ with respect to the free variables, subject to the equality constraints. With this definition, the quantities $w_{j}+q_{j}$ are the Lagrange multipliers at the minimizer $x_{j}+p_{j}$. The components of $p_{j}$ with indices in $\mathcal{A}\left(x_{j}\right)$ are zero, and the free components $p_{F}=\left[p_{j}\right]_{F}$ are determined from the equations

$$
\left(\begin{array}{cc}
H_{F} & -A_{F}^{T} \\
A_{F} & 0
\end{array}\right)\left(\begin{array}{l}
p_{F} \\
q_{j}
\end{array}\right)=-\left(\begin{array}{c}
{\left[g+H\left(x_{j}-\bar{x}\right)-A^{T} w_{j}\right]_{F}} \\
c+A\left(x_{j}-\bar{x}\right)
\end{array}\right)
$$

where $[\cdot]_{F}$ denotes the subvector of components with indices in $\mathcal{F}\left(x_{j}\right)$. The choice of step length $\alpha_{j}$ is based on remaining feasible with respect to the satisfied bounds. If $x_{j}+p_{j}$ is feasible, i.e., $x_{j}+p_{j} \geq 0$, then $\alpha_{j}$ will be taken as unity. Otherwise, $\alpha$ is set to $\alpha_{M}$, the largest feasible step along $p_{j}$. Finally, the iteration index $j$ is incremented by one and the iteration is repeated.

It must be emphasized that this active-set method is not well defined unless the equations (5.2) have a solution at every $\left(x_{j}, w_{j}\right)$.

\subsection{Solution of the bound-constrained subproblem}

Next we apply the active-set method to a bound constrained QP of the form

$$
\min _{v} \nabla M^{T}(v-\bar{v})+\frac{1}{2}(v-\bar{v})^{T} B^{\nu}(v-\bar{v}) \quad \text { subject to } v_{i} \geq 0, i=1,2, \ldots, n,
$$

where $v$ is the vector of $n+m$ primal-dual variables $v=(x, y), \bar{v}$ is the constant vector $\bar{v}=(\bar{x}, \bar{y})$, and

$$
\nabla M=\left(\begin{array}{c}
g-J^{T}(\pi+\nu(\pi-\bar{y})) \\
\nu\left(c+\mu\left(\bar{y}-y^{E}\right)\right)
\end{array}\right), \quad B^{\nu}=\left(\begin{array}{cc}
\widehat{H}+\frac{1}{\mu}(1+\nu) J^{T} J & \nu J^{T} \\
\nu J & \nu \mu I
\end{array}\right)
$$

where $\widehat{H}$ is chosen so that $\widehat{H}+\frac{1}{\mu} J^{T} J$ is positive definite. (See Section 4 for additional details.) It follows from Lemma 2.1 that the bound constrained problem (5.3) is a convex QP that may be solved using the conventional active-set method 
of Section 5.1. At the $j$ th iterate $v_{j}=\left(x_{j}, y_{j}\right)$, the index sets of active and free variables are given by $\widehat{\mathcal{A}}\left(v_{j}\right)$ and $\widehat{\mathcal{F}}\left(v_{j}\right)$, where

$$
\widehat{\mathcal{A}}(v)=\mathcal{A}(x)=\left\{i: x_{i}=0\right\} \quad \text { and } \quad \widehat{\mathcal{F}}(v)=\{1,2, \ldots, n+m\} \backslash \widehat{\mathcal{A}}(v)
$$

(cf. (1.3)). (As the dual variables are not subject to bounds, the vector of free components of any $v=(x, y)$ has the form $v_{\widehat{F}}=\left(x_{F}, y\right)$ with $x_{F}$ defined in terms of $\mathcal{F}$.) Given $v_{j}=\left(x_{j}, y_{j}\right)$, the next QP iterate is defined as $v_{j+1}=v_{j}+\alpha_{j} d_{j}$, where the free components of the vector $d_{j}=\left(p_{j}, q_{j}\right)$ satisfy the equations

$$
B_{\widehat{F}}^{\nu} d_{\widehat{F}}=-\left[\nabla M+B^{\nu}\left(v_{j}-\bar{v}\right)\right]_{\widehat{F}},
$$

with $d_{\widehat{F}}=\left(p_{F}, q_{j}\right)$. The equations (5.4) appear to be ill-conditioned for small $\mu$ because of the $O(1 / \mu)$ term in the $(1,1)$ block of the matrix $B^{\nu}$. However, this ill-conditioning is superficial. The next result shows that $d_{F}$ may be determined by solving an equivalent nonsingular primal-dual system with conditioning dependent on that of the original problem.

Theorem 5.1. Consider the application of the active-set method to the bound constrained $Q P$ (5.3). Then, for every $\nu \geq 0$, the free components of the QP search direction $\left(p_{j}, q_{j}\right)$ satisfy the nonsingular primal-dual system

$$
\left(\begin{array}{cc}
\widehat{H}_{F} & -J_{F}^{T} \\
J_{F} & \mu I
\end{array}\right)\left(\begin{array}{l}
p_{F} \\
q_{j}
\end{array}\right)=-\left(\begin{array}{c}
{\left[g+\widehat{H}\left(x_{j}-\bar{x}\right)-J^{T} y_{j}\right]_{F}} \\
c+\mu\left(y_{j}-y^{E}\right)+J\left(x_{j}-\bar{x}\right)
\end{array}\right) .
$$

Proof. Consider the definition of the search direction when $\nu>0$. In this case it suffices to show that the linear systems (5.4) and (5.5) are equivalent. For any positive $\nu$, we may define the matrix

$$
T=\left(\begin{array}{cc}
I & -\frac{1+\nu}{\nu \mu} J_{F}^{T} \\
0 & \frac{1}{\nu} I_{m}
\end{array}\right),
$$

where the identity matrix $I$ has dimension $n_{F}$, the column dimension of $J_{F}$. The matrix $T$ is nonsingular with $n_{F}+m$ rows and columns. It follows that the equations

$$
T B_{\widehat{F}}^{\nu} d_{\widehat{F}}=-T\left[\nabla M+B^{\nu}\left(v_{j}-\bar{v}\right)\right]_{\widehat{F}}
$$

have the same solution as those of (5.4). The primal-dual equations (5.5) follow by direct multiplication. The nonsingularity of the equations (5.5) follows from the nonsingularity of $T$, and the fact that $B^{\nu}$ is positive definite (as are all symmetric submatrices formed from its rows and columns).

The resulting equations (5.5) are independent of $\nu$, but the proof above is not applicable when $\nu=0$ because $T$ is undefined in this case. For $\nu=0$, the QP objective includes only the primal variables $x$, which implies that problem (5.3) may be written as

$$
\underset{x \geq 0}{\operatorname{minimize}}\left(g-J^{T} \pi\right)^{T}(x-\bar{x})+\frac{1}{2}(x-\bar{x})^{T}\left(\widehat{H}+\frac{1}{\mu} J^{T} J\right)(x-\bar{x}),
$$


with $y$ arbitrary. The active-set equations analogous to (5.4) are then

$$
\left(\widehat{H}_{F}+\frac{1}{\mu} J_{F}^{T} J_{F}\right) p_{F}=-\left[g+\left(\widehat{H}+\frac{1}{\mu} J^{T} J\right)\left(x_{j}-\bar{x}\right)-J^{T} \pi\right]_{F} .
$$

For any choice of $y_{j}$, define the $m$-vector $q_{j}$ such that

$$
q_{j}=-\frac{1}{\mu}\left(J_{F} p_{F}+\mu\left(y_{j}-\pi\right)+J\left(x_{j}-\bar{x}\right)\right),
$$

where $\pi=y^{E}-c / \mu$ (see (2.3)). Equations (5.6) and (5.7) may be combined to give equations $K d_{\widehat{F}}=-r$, where $d_{\widehat{F}}=\left(p_{F}, q_{j}\right)$,

$$
K=\left(\begin{array}{cc}
\widehat{H}_{F}+\frac{2}{\mu} J_{F}^{T} J_{F} & J_{F}^{T} \\
J_{F} & \mu I
\end{array}\right)
$$

and the right-hand side is

$$
r=\left(\begin{array}{c}
{\left[g+\widehat{H}\left(x_{j}-\bar{x}\right)\right]_{F}+\frac{2}{\mu} J_{F}^{T} J\left(x_{j}-\bar{x}\right)-J_{F}^{T} y_{j}+2 J_{F}^{T}\left(y_{j}-\pi\right)} \\
\mu\left(y_{j}-\pi\right)+J\left(x_{j}-\bar{x}\right)
\end{array}\right) .
$$

Forming the equations $T K d_{\widehat{F}}=-T r$, where $T$ is the nonsingular matrix

$$
T=\left(\begin{array}{cc}
I & -\frac{2}{\mu} J_{F}^{T} \\
0 & I_{m}
\end{array}\right)
$$

gives the equivalent system

$$
\left(\begin{array}{cc}
\widehat{H}_{F} & -J_{F}^{T} \\
J_{F} & \mu I
\end{array}\right)\left(\begin{array}{c}
p_{F} \\
q_{j}
\end{array}\right)=-\left(\begin{array}{c}
{\left[g+\widehat{H}\left(x_{j}-\bar{x}\right)-J^{T} y_{j}\right]_{F}} \\
c+\mu\left(y_{j}-y^{E}\right)+J\left(x_{j}-\bar{x}\right)
\end{array}\right)
$$

which is identical to the system (5.5).

\subsection{Solution of the stabilized SQP subproblem}

Consider the application of the conventional active-set method of Section 5.1 to the stabilized QP:

$$
\begin{array}{cl}
\underset{x, y}{\operatorname{minimize}} & g^{T}(x-\bar{x})+\frac{1}{2}(x-\bar{x})^{T} \widehat{H}(x-\bar{x})+\frac{1}{2} \mu\|y\|^{2} \\
\text { subject to } & c+J(x-\bar{x})+\mu\left(y-y^{E}\right)=0, \quad x \geq 0
\end{array}
$$

In terms of the data " $(x, \bar{x}, H, g, A, c)$ " for the generic QP (5.1), we have variables $" x "=(x, y)$, with " $\bar{x} "=(\bar{x}, \bar{y})$,

$$
" H "=\left(\begin{array}{cc}
\widehat{H} & 0 \\
0 & \mu I
\end{array}\right), \quad " g "=\left(\begin{array}{c}
g \\
\mu \bar{y}
\end{array}\right), \quad " A "=\left(\begin{array}{ll}
J & \mu I
\end{array}\right), \text { and } " c "=c+\mu\left(\bar{y}-y^{E}\right) \text {. }
$$


(The discussion of the properties of the stabilized QP relative to the generic form (5.1) is not affected by the nonnegativity constraints being applied to only a subset of the variables in (5.8).) After some simplification, the equations analogous to (5.2) may be written as

$$
\left(\begin{array}{ccc}
\widehat{H}_{F} & 0 & -J_{F}^{T} \\
0 & \mu I & -\mu I \\
J_{F} & \mu I & 0
\end{array}\right)\left(\begin{array}{c}
p_{F} \\
\bar{p}_{F} \\
q_{j}
\end{array}\right)=-\left(\begin{array}{c}
{\left[g+\widehat{H}\left(x_{j}-\bar{x}\right)-J^{T} w_{j}\right]_{F}} \\
\mu y_{j}-\mu w_{j} \\
c+\mu\left(y_{j}-y^{E}\right)+J\left(x_{j}-\bar{x}\right)
\end{array}\right),
$$

where $p_{F}$ and $\bar{p}_{F}$ denote the free components of the search directions for the $x$ and $y$ variables respectively. (Observe that the right-hand side of (5.9) is independent of $\bar{y}$.) The second block of equations gives $\bar{p}_{F}=q_{j}-y_{j}+w_{j}$, which implies that

$$
y_{j+1}=y_{j}+\bar{p}_{F}=y_{j}+q_{j}-y_{j}+w_{j}=w_{j}+q_{j}=w_{j+1},
$$

so that the primal $y$-variables and dual variables of the stabilized QP are identical.

Similarly, substituting for $\bar{p}_{F}$ in the third block of equations in (5.10), and using the primal-dual equivalence $w_{j}=y_{j}$ gives

$$
\left(\begin{array}{cc}
\widehat{H}_{F} & -J_{F}^{T} \\
J_{F} & \mu I
\end{array}\right)\left(\begin{array}{l}
p_{F} \\
q_{j}
\end{array}\right)=-\left(\begin{array}{c}
{\left[g+\widehat{H}\left(x_{j}-\bar{x}\right)-J^{T} y_{j}\right]_{F}} \\
c+\mu\left(y_{j}-y^{E}\right)+J\left(x_{j}-\bar{x}\right)
\end{array}\right)
$$

which are identical to the equations associated with those for the QP subproblem (5.3).

The preceding discussion constitutes a proof of the following result.

Theorem 5.2. Consider the application of the active-set method to the bound constrained QP (5.3) and stabilized $Q P(5.8)$ defined with the same quantities $c, g, J$ and $\widehat{H}$. Consider any $x_{0}$ and $y_{0}$ such that $\left(x_{0}, y_{0}\right)$ is feasible for the stabilized $Q P$ (5.8). Then, for every $\nu \geq 0$, the active-set method generates identical primal-dual iterates $\left\{\left(x_{j}, y_{j}\right)\right\}_{j \geq 0}$.

\section{Numerical Results}

Numerical results from a simple MATLAB implementation of pdSQP (Algorithm 2.1) were obtained for 158 problems from the CUTEr test collection (see Bongartz et al. [2], and Gould, Orban and Toint [28]). The problems tested were the nonlinearly constrained problems from the Hock and Schittkowski [34] test suite, and the CUTEr equality constrained problems considered in [23]. A total of 15 problems were excluded from the Hock and Schittkowski set. Problems hs1, hs2, hs3, hs3mod, hs4, hs5, hs25, hs38, hs45 and hs110 have no general constraints; problems hs67, hs85

and hs87 are nonsmooth; the objective function of hs84 is unbounded in the feasible region; and problem hs99exp is poorly scaled.

Each CUTEr problem may be written in the form

$$
\underset{x}{\operatorname{minimize}} f(x) \quad \text { subject to }\left(\begin{array}{l}
x_{l} \\
c_{l}
\end{array}\right) \leq\left(\begin{array}{c}
x \\
C(x)
\end{array}\right) \leq\left(\begin{array}{c}
x_{u} \\
c_{u}
\end{array}\right),
$$


where $C: \mathbb{R}^{n} \mapsto \mathbb{R}^{m}, f: \mathbb{R}^{n} \mapsto \mathbb{R}$, and $\left(x_{l}, c_{l}\right)$ and $\left(x_{u}, c_{u}\right)$ are constant vectors of lower and upper bounds. In this format, a fixed variable or equality constraint has the same value for its upper and lower bound. For pdSQP, each problem was converted to the equivalent form

$$
\underset{x, s}{\operatorname{minimize}} f(x) \quad \text { subject to } c(x, s)=C(x)-s=0, \quad\left(\begin{array}{l}
x_{l} \\
c_{l}
\end{array}\right) \leq\left(\begin{array}{l}
x \\
s
\end{array}\right) \leq\left(\begin{array}{l}
x_{u} \\
c_{u}
\end{array}\right),
$$

where $s$ is a vector of slack variables. With this formulation, the bound constrained and stabilized QP subproblems involve simple upper and lower bounds instead of nonnegativity constraints. The MATLAB implementation was initialized with parameter values given in Table 1 . The primal-dual vector $\left(x_{0}, y_{0}\right)$ was the default values supplied by CUTEr, although the code immediately projects $x_{0}$ to ensure feasibility with respect to the simple bounds on $x$. The sequence of iterates was terminated at a point satisfying the condition

$$
\left\|r_{\mathrm{opt}}\left(v_{k}\right)\right\|_{\infty}<\tau_{\text {stop }}
$$

where $r_{\mathrm{opt}}\left(v_{k}\right)$ is the optimality measure (2.23) defined in terms of the problem format (6.2).

Table 1: Control parameters and initial values for Algorithm pdSQP.

\begin{tabular}{|cl|cl|cl|}
\hline Parameter & Value & Parameter & Value & Parameter & Value \\
\hline$\nu$ & 1.0 & $\alpha_{\min }$ & $1.0 \mathrm{e}-3$ & $\mu_{0}^{R}$ & $1.0 \mathrm{e}-4$ \\
$y_{\max }$ & $1.0 \mathrm{e}+6$ & $\tau_{\text {stop }}$ & $1.0 \mathrm{e}-6$ & $\mu_{0}$ & 1.0 \\
$\eta_{S}$ & $1.0 \mathrm{e}-2$ & $k_{\max }$ & 600 & $\tau_{0}$ & $1.0 \mathrm{e}-2$ \\
$\eta_{D}$ & $1.0 \mathrm{e}-3$ & $\beta$ & $1.0 \mathrm{e}-5$ & $\phi_{V}^{\max }, \phi_{O}^{\max }$ & $1.0 \mathrm{e}+3$ \\
\hline
\end{tabular}

Algorithm pdSQP solves the bound constrained QP subproblem (2.8) using a MATLAB version of the inertia-controlling QP solver of Gill and Wong [26], which solves a sequence of perturbed KKT systems of the form (2.9). At the start of each subproblem, an initial QP working set is defined that contains the indices of all the fixed variables and variables within $\epsilon_{A}=10^{-6}$ of their upper or lower bounds. This set is used to compute the matrix $H_{F}$ and the (possibly) modified Hessian $\widehat{H}=H+E+D$ used in (2.6). In some situations, it is possible to guarantee that both $E$ and $D$ are zero, i.e., no convexification is needed. If the problem (6.1) is a convex program then it can be shown that the matrix $B^{\nu}$ is positive definite with $\widehat{H}=H$ (see Kungurtsev [37]). Similarly, the elements of $H$ corresponding to fixed variables need not be modified because the corresponding elements of $d$ are always zero. This implies that $D \equiv 0$ for problems with only equality constraints (i.e., problems such that $c_{l}=c_{u}$ for the bound constraints of (6.2)). In pdSQP, the matrix $\widehat{H}$ is defined as described in Section 4, where the matrix $E_{F}$ of (4.2) is determined using the inertia controlling $\mathrm{LBL}^{T}$ factorization (see, $[17,18]$ for more details). For the calculation of the modification $D=\left(1 / \mu_{A}\right) P_{A} P_{A}^{T}$, we consider the properties of 
the matrix

$$
K_{F A}=\left(\begin{array}{cc}
H_{F A}+E_{F A} & J_{F A}^{T} \\
J_{F A} & -\mu_{A} I_{m}
\end{array}\right),
$$

where $H_{F A}$ and $E_{F A}$ denote the $n_{F A} \times n_{F A}$ rows and columns of $H$ and $E$ associated with the union of the index sets of free variables and active bound constraints (i.e., fixed variables are not included). A similar definition holds for the matrix $J_{F A}$ formed from a subset of the columns of $J$. Given the value

$$
\sigma=\min \left(10^{-1}, 1 / \max (1,\|E\|)\right),
$$

the parameter $\mu_{A}$ is then the first member of the sequence $\sigma, \sigma / 10, \sigma / 10^{2}, \ldots$, for which $K_{F A}$ has inertia $\left(n_{F A}, m, 0\right)$. (Theorem 4.1 implies that this sequence must terminate.) This method is clearly impractical for any serious implementation. The discussion of more efficient methods that compute the modified matrix during the solution of the QP subproblem are beyond the scope of this paper (see Kungurtsev [37]). We recognize that other definitions of $\widehat{H}$ are possible, including a positive-definite quasi-Newton approximations based on the BFGS update (see, e.g., $[21,42])$.

Table 2 gives the results of running algorithm pdSQP on the 158 CUTEr test problems. For each problem the table lists the number of general constraint functions (" $m$ "), the number of $x$ variables (" $n$ "), the number of function evaluations ("fe"), and the number of outer iterations ("itns"). A run is considered to have failed if pdSQP could not satisfy the optimality condition (6.3) in $k_{\max }=600$ iterations. The function and iteration entries for these "failed" runs are marked with an "F". The columns with headings " $\|E\|>0$ " and " $\|D\|>0$ " give the percentage of iterations for which the Hessian modifications $E$ and $D$ of (4.1) were nonzero. The columns "VO-itns" and "F-itns" give the percentages of V-or O-iterates and Fiterates required (see Algorithm 2.1). The column with heading "M-itns" gives the total number of M-iterates required for each problem. (The M-iterates are not listed as a percentage of the total number of iterations because they generally constitute significantly less than $1 \%$ of the total iterations.)

Algorithm pdSQP was able to satisfy the optimality measure for 150 of the 158 test problems. Of the 8 "failures", the final point for lukvle8 satisfied the complementarity measure but gave a maximum constraint violation of $1.1 \times 10^{-4}$. The problem can be solved successfully in 771 iterations, of which $22 \%$ are $\mathrm{V}$ - or Oiterates, $76 \%$ are F-itns and 8 iterations are M-iterates. Problem mss 1 can be solved in 2872 iterations with F-iterates forming $92 \%$ of the total iterations. In this case, $99 \%$ of the iterations required some form of convexification. The six problems: dixchlng, hs106, hs109, hs116, lukvle6, and lukvle14 terminated at infeasible local minimizers of the merit function, as measured by the optimality conditions for the infeasibility problem

$$
\underset{x, s}{\operatorname{minimize}} \frac{1}{2}\|c(x, s)\|_{2}^{2} \quad \text { subject to }\left(\begin{array}{l}
x_{l} \\
c_{l}
\end{array}\right) \leq\left(\begin{array}{l}
x \\
s
\end{array}\right) \leq\left(\begin{array}{l}
x_{u} \\
c_{u}
\end{array}\right) .
$$

The CUTEr collection includes problems with a wide range of difficulty. However, there are groups of problems that are variants of one case (see, e.g., the problems 
hs88-hs92 and hs101-hs103). Typically, a method will behave in a similar way on all the problems of one type, which can distort the results of numerical tests. These observations notwithstanding, the results of Table 2 indicate that pdSQP is robust overall, and that the primal-dual augmented Lagrangian provides an effective way of ensuring global convergence. For the 150 problems that were solved successfully, a grand total of $94 \%$ of the iterations computed a V- or O-iterate, and $5 \%$ of the iterations computed an F-iterate. An M-iterate was computed in only 26 of the iterations required to solve all 150 problems.

The results illustrate the importance of an effective convexification strategy. Overall, 77 of the 158 problems required some form of convexification. Of these 77 problems, a grand total of $44 \%$ of the iterations required the computation of a nonzero $E$, and $23 \%$ of the iterations required a nonzero $D$.

Table 2: Results for pdSQP on a subset of the CUTEr test set

\begin{tabular}{|c|c|c|c|c|c|c|c|c|c|}
\hline Problem & $m$ & $n$ & fe & itns & $\|E\|>0$ & $\|D\|>0$ & VO-itns & F-itns & M-itns \\
\hline bt1 & 1 & 2 & 27 & 14 & $0 \%$ & $0 \%$ & $100 \%$ & $0 \%$ & 0 \\
\hline bt2 & 1 & 3 & 15 & 11 & $0 \%$ & $0 \%$ & $100 \%$ & $0 \%$ & 0 \\
\hline bt3 & 3 & 5 & 5 & 4 & $0 \%$ & $0 \%$ & $100 \%$ & $0 \%$ & 0 \\
\hline bt4 & 2 & 3 & 31 & 15 & $26 \%$ & $0 \%$ & $100 \%$ & $0 \%$ & 0 \\
\hline bt5 & 2 & 3 & 24 & 10 & $30 \%$ & $0 \%$ & $100 \%$ & $0 \%$ & 0 \\
\hline bt6 & 2 & 5 & 33 & 20 & $20 \%$ & $0 \%$ & $100 \%$ & $0 \%$ & 0 \\
\hline bt7 & 3 & 5 & 63 & 42 & $4 \%$ & $0 \%$ & $100 \%$ & $0 \%$ & 0 \\
\hline bt8 & 2 & 5 & 12 & 11 & $81 \%$ & $0 \%$ & $100 \%$ & $0 \%$ & 0 \\
\hline bt 9 & 2 & 4 & 24 & 14 & $0 \%$ & $0 \%$ & $100 \%$ & $0 \%$ & 0 \\
\hline bt10 & 2 & 2 & 7 & 6 & $0 \%$ & $0 \%$ & $100 \%$ & $0 \%$ & 0 \\
\hline bt11 & 3 & 5 & 14 & 8 & $0 \%$ & $0 \%$ & $100 \%$ & $0 \%$ & 0 \\
\hline bt12 & 3 & 5 & 14 & 6 & $0 \%$ & $0 \%$ & $100 \%$ & $0 \%$ & 0 \\
\hline byrdsphr & 2 & 3 & 36 & 17 & $52 \%$ & $0 \%$ & $100 \%$ & $0 \%$ & 0 \\
\hline coolhans & 9 & 9 & 45 & 41 & $39 \%$ & $0 \%$ & $86 \%$ & $14 \%$ & 0 \\
\hline dixchlng & 5 & 10 & $\mathrm{~F}$ & $\mathrm{~F}$ & $0 \%$ & $0 \%$ & $17 \%$ & $83 \%$ & 1 \\
\hline eigena2 & 55 & 110 & 9 & 5 & $0 \%$ & $0 \%$ & $100 \%$ & $0 \%$ & 0 \\
\hline eigenaco & 55 & 110 & 9 & 5 & $0 \%$ & $0 \%$ & $100 \%$ & $0 \%$ & 0 \\
\hline eigenb2 & 55 & 110 & 239 & 117 & $93 \%$ & $0 \%$ & $25 \%$ & $75 \%$ & 0 \\
\hline eigenbco & 55 & 110 & 72 & 37 & $43 \%$ & $0 \%$ & $100 \%$ & $0 \%$ & 0 \\
\hline eigenc2 & 231 & 462 & 33 & 15 & $66 \%$ & $0 \%$ & $100 \%$ & $0 \%$ & 0 \\
\hline eigencco & 231 & 462 & 88 & 46 & $58 \%$ & $0 \%$ & $72 \%$ & $28 \%$ & 0 \\
\hline elec & 200 & 600 & 100 & 50 & $86 \%$ & $0 \%$ & $88 \%$ & $12 \%$ & 0 \\
\hline gridnete & 36 & 60 & 5 & 4 & $0 \%$ & $0 \%$ & $100 \%$ & $0 \%$ & 0 \\
\hline gridneth & 36 & 60 & 12 & 11 & $0 \%$ & $0 \%$ & $100 \%$ & $0 \%$ & 0 \\
\hline hs 6 & 1 & 2 & 27 & 11 & $0 \%$ & $0 \%$ & $100 \%$ & $0 \%$ & 0 \\
\hline hs7 & 1 & 2 & 30 & 14 & $57 \%$ & $0 \%$ & $100 \%$ & $0 \%$ & 0 \\
\hline hs8 & 2 & 2 & 6 & 4 & $0 \%$ & $0 \%$ & $100 \%$ & $0 \%$ & 0 \\
\hline hs9 & 1 & 2 & 6 & 4 & $0 \%$ & $0 \%$ & $100 \%$ & $0 \%$ & 0 \\
\hline $\mathrm{hs} 10$ & 1 & 2 & 15 & 12 & $0 \%$ & $0 \%$ & $100 \%$ & $0 \%$ & 0 \\
\hline hs11 & 1 & 2 & 7 & 6 & $0 \%$ & $0 \%$ & $100 \%$ & $0 \%$ & 0 \\
\hline hs 12 & 1 & 2 & 15 & 7 & $0 \%$ & $0 \%$ & $100 \%$ & $0 \%$ & 0 \\
\hline
\end{tabular}


Table 2: Results for CUTEr test problems (continued)

\begin{tabular}{|c|c|c|c|c|c|c|c|c|c|}
\hline Problem & $m$ & $n$ & fe & itns & $\|E\|>0$ & $\|D\|>0$ & VO-itns & F-itns & M-itns \\
\hline $\mathrm{hs} 13$ & 2 & 2 & 81 & 78 & $2 \%$ & $5 \%$ & $93 \%$ & $7 \%$ & 0 \\
\hline hs14 & 2 & 2 & 14 & 6 & $0 \%$ & $0 \%$ & $100 \%$ & $0 \%$ & 0 \\
\hline hs15 & 2 & 2 & 340 & 339 & $50 \%$ & $53 \%$ & $19 \%$ & $81 \%$ & 1 \\
\hline hs16 & 2 & 2 & 7 & 5 & $0 \%$ & $80 \%$ & $100 \%$ & $0 \%$ & 0 \\
\hline hs 17 & 2 & 2 & 11 & 9 & $11 \%$ & $11 \%$ & $100 \%$ & $0 \%$ & 0 \\
\hline hs18 & 2 & 2 & 16 & 9 & $0 \%$ & $22 \%$ & $100 \%$ & $0 \%$ & 0 \\
\hline hs19 & 2 & 2 & 18 & 17 & $29 \%$ & $17 \%$ & $100 \%$ & $0 \%$ & 0 \\
\hline hs20 & 3 & 2 & 4 & 3 & $0 \%$ & $66 \%$ & $100 \%$ & $0 \%$ & 0 \\
\hline hs21 & 1 & 2 & 8 & 2 & $0 \%$ & $0 \%$ & $100 \%$ & $0 \%$ & 0 \\
\hline hs 21 mod & 1 & 7 & 8 & 2 & $0 \%$ & $0 \%$ & $100 \%$ & $0 \%$ & 0 \\
\hline hs22 & 2 & 2 & 12 & 5 & $0 \%$ & $0 \%$ & $100 \%$ & $0 \%$ & 0 \\
\hline hs23 & 5 & 2 & 17 & 9 & $22 \%$ & $55 \%$ & $100 \%$ & $0 \%$ & 0 \\
\hline hs24 & 3 & 2 & 4 & 3 & $0 \%$ & $66 \%$ & $100 \%$ & $0 \%$ & 0 \\
\hline hs 26 & 1 & 3 & 28 & 20 & $0 \%$ & $0 \%$ & $100 \%$ & $0 \%$ & 0 \\
\hline hs27 & 1 & 3 & 25 & 13 & $15 \%$ & $0 \%$ & $100 \%$ & $0 \%$ & 0 \\
\hline hs28 & 1 & 3 & 9 & 3 & $0 \%$ & $0 \%$ & $100 \%$ & $0 \%$ & 0 \\
\hline hs29 & 1 & 3 & 16 & 8 & $62 \%$ & $25 \%$ & $100 \%$ & $0 \%$ & 0 \\
\hline hs30 & 1 & 3 & 15 & 11 & $0 \%$ & $0 \%$ & $100 \%$ & $0 \%$ & 0 \\
\hline hs31 & 1 & 3 & 10 & 6 & $0 \%$ & $50 \%$ & $100 \%$ & $0 \%$ & 0 \\
\hline hs32 & 2 & 3 & 9 & 5 & $0 \%$ & $0 \%$ & $100 \%$ & $0 \%$ & 0 \\
\hline hs33 & 2 & 3 & 6 & 4 & $25 \%$ & $75 \%$ & $100 \%$ & $0 \%$ & 0 \\
\hline hs34 & 2 & 3 & 12 & 6 & $50 \%$ & $33 \%$ & $100 \%$ & $0 \%$ & 0 \\
\hline hs35 & 1 & 3 & 5 & 3 & $0 \%$ & $0 \%$ & $100 \%$ & $0 \%$ & 0 \\
\hline hs35i & 1 & 3 & 5 & 3 & $0 \%$ & $0 \%$ & $100 \%$ & $0 \%$ & 0 \\
\hline hs 35 mod & 1 & 3 & 3 & 2 & $0 \%$ & $0 \%$ & $100 \%$ & $0 \%$ & 0 \\
\hline hs 36 & 1 & 3 & 4 & 3 & $0 \%$ & $66 \%$ & $100 \%$ & $0 \%$ & 0 \\
\hline hs37 & 2 & 3 & 7 & 6 & $0 \%$ & $83 \%$ & $100 \%$ & $0 \%$ & 0 \\
\hline hs39 & 2 & 4 & 24 & 14 & $0 \%$ & $0 \%$ & $100 \%$ & $0 \%$ & 0 \\
\hline hs 40 & 3 & 4 & 11 & 5 & $0 \%$ & $0 \%$ & $100 \%$ & $0 \%$ & 0 \\
\hline hs41 & 1 & 4 & 7 & 6 & $0 \%$ & $83 \%$ & $100 \%$ & $0 \%$ & 0 \\
\hline hs42 & 2 & 4 & 14 & 6 & $0 \%$ & $0 \%$ & $100 \%$ & $0 \%$ & 0 \\
\hline hs 43 & 3 & 4 & 15 & 7 & $0 \%$ & $0 \%$ & $100 \%$ & $0 \%$ & 0 \\
\hline hs44 & 6 & 4 & 10 & 8 & $75 \%$ & $50 \%$ & $100 \%$ & $0 \%$ & 0 \\
\hline hs 44 new & 6 & 4 & 10 & 7 & $71 \%$ & $28 \%$ & $100 \%$ & $0 \%$ & 0 \\
\hline hs 46 & 2 & 5 & 25 & 18 & $5 \%$ & $0 \%$ & $100 \%$ & $0 \%$ & 0 \\
\hline hs 47 & 3 & 5 & 23 & 15 & $0 \%$ & $0 \%$ & $100 \%$ & $0 \%$ & 0 \\
\hline hs 48 & 2 & 5 & 7 & 2 & $0 \%$ & $0 \%$ & $100 \%$ & $0 \%$ & 0 \\
\hline hs 49 & 2 & 5 & 14 & 9 & $0 \%$ & $0 \%$ & $100 \%$ & $0 \%$ & 0 \\
\hline hs50 & 3 & 5 & 14 & 10 & $0 \%$ & $0 \%$ & $100 \%$ & $0 \%$ & 0 \\
\hline hs51 & 3 & 5 & 8 & 2 & $0 \%$ & $0 \%$ & $100 \%$ & $0 \%$ & 0 \\
\hline hs52 & 3 & 5 & 7 & 3 & $0 \%$ & $0 \%$ & $100 \%$ & $0 \%$ & 0 \\
\hline hs53 & 3 & 5 & 4 & 3 & $0 \%$ & $0 \%$ & $100 \%$ & $0 \%$ & 0 \\
\hline hs54 & 1 & 6 & 3 & 2 & $50 \%$ & $50 \%$ & $100 \%$ & $0 \%$ & 0 \\
\hline hs55 & 6 & 6 & 3 & 2 & $0 \%$ & $50 \%$ & $100 \%$ & $0 \%$ & 0 \\
\hline
\end{tabular}


Table 2: Results for CUTEr test problems (continued)

\begin{tabular}{|c|c|c|c|c|c|c|c|c|c|}
\hline Problem & $m$ & $n$ & fe & itns & $\|E\|>0$ & $\|D\|>0$ & VO-itns & F-itns & M-itns \\
\hline hs56 & 4 & 7 & 68 & 61 & $72 \%$ & $0 \%$ & $87 \%$ & $13 \%$ & 0 \\
\hline hs57 & 1 & 2 & 8 & 3 & $66 \%$ & $0 \%$ & $100 \%$ & $0 \%$ & 0 \\
\hline hs59 & 3 & 2 & 151 & 77 & $96 \%$ & $2 \%$ & $43 \%$ & $57 \%$ & 0 \\
\hline hs 60 & 1 & 3 & 8 & 6 & $0 \%$ & $0 \%$ & $100 \%$ & $0 \%$ & 0 \\
\hline hs 61 & 2 & 3 & 14 & 7 & $0 \%$ & $0 \%$ & $100 \%$ & $0 \%$ & 0 \\
\hline hs 62 & 1 & 3 & 69 & 68 & $0 \%$ & $0 \%$ & $78 \%$ & $22 \%$ & 0 \\
\hline hs 63 & 2 & 3 & 68 & 43 & $55 \%$ & $0 \%$ & $96 \%$ & $4 \%$ & 0 \\
\hline hs 64 & 1 & 3 & 474 & 473 & $0 \%$ & $0 \%$ & $18 \%$ & $80 \%$ & 3 \\
\hline hs 65 & 1 & 3 & 21 & 11 & $0 \%$ & $0 \%$ & $100 \%$ & $0 \%$ & 0 \\
\hline hs 66 & 2 & 3 & 10 & 5 & $40 \%$ & $40 \%$ & $100 \%$ & $0 \%$ & 0 \\
\hline hs 68 & 2 & 4 & 30 & 28 & $25 \%$ & $53 \%$ & $100 \%$ & $0 \%$ & 0 \\
\hline hs 69 & 2 & 4 & 15 & 9 & $22 \%$ & $0 \%$ & $100 \%$ & $0 \%$ & 0 \\
\hline hs 70 & 1 & 4 & 21 & 15 & $20 \%$ & $0 \%$ & $100 \%$ & $0 \%$ & 0 \\
\hline hs71 & 2 & 4 & 6 & 5 & $0 \%$ & $20 \%$ & $100 \%$ & $0 \%$ & 0 \\
\hline hs72 & 2 & 4 & 463 & 459 & $0 \%$ & $0 \%$ & $30 \%$ & $67 \%$ & 10 \\
\hline hs73 & 3 & 4 & 4 & 3 & $0 \%$ & $0 \%$ & $100 \%$ & $0 \%$ & 0 \\
\hline hs74 & 5 & 4 & 14 & 13 & $0 \%$ & $0 \%$ & $100 \%$ & $0 \%$ & 0 \\
\hline hs 75 & 5 & 4 & 49 & 48 & $0 \%$ & $0 \%$ & $100 \%$ & $0 \%$ & 0 \\
\hline hs76 & 3 & 4 & 4 & 3 & $0 \%$ & $0 \%$ & $100 \%$ & $0 \%$ & 0 \\
\hline hs76i & 3 & 4 & 4 & 3 & $0 \%$ & $0 \%$ & $100 \%$ & $0 \%$ & 0 \\
\hline hs77 & 2 & 5 & 21 & 16 & $0 \%$ & $0 \%$ & $100 \%$ & $0 \%$ & 0 \\
\hline hs78 & 3 & 5 & 14 & 6 & $0 \%$ & $0 \%$ & $100 \%$ & $0 \%$ & 0 \\
\hline hs79 & 3 & 5 & 15 & 8 & $0 \%$ & $0 \%$ & $100 \%$ & $0 \%$ & 0 \\
\hline hs 80 & 3 & 5 & 6 & 5 & $0 \%$ & $0 \%$ & $100 \%$ & $0 \%$ & 0 \\
\hline hs81 & 3 & 5 & 7 & 6 & $0 \%$ & $0 \%$ & $100 \%$ & $0 \%$ & 0 \\
\hline hs83 & 3 & 5 & 11 & 10 & $80 \%$ & $90 \%$ & $100 \%$ & $0 \%$ & 0 \\
\hline hs 86 & 10 & 5 & 5 & 3 & $0 \%$ & $0 \%$ & $100 \%$ & $0 \%$ & 0 \\
\hline hs88 & 1 & 2 & 92 & 84 & $0 \%$ & $0 \%$ & $97 \%$ & $0 \%$ & 2 \\
\hline hs89 & 1 & 3 & 95 & 87 & $0 \%$ & $1 \%$ & $97 \%$ & $0 \%$ & 2 \\
\hline hs 90 & 1 & 4 & 94 & 85 & $5 \%$ & $1 \%$ & $97 \%$ & $0 \%$ & 2 \\
\hline hs91 & 1 & 5 & 99 & 86 & $12 \%$ & $2 \%$ & $96 \%$ & $0 \%$ & 3 \\
\hline hs92 & 1 & 6 & 105 & 91 & $13 \%$ & $1 \%$ & $97 \%$ & $0 \%$ & 2 \\
\hline hs93 & 2 & 6 & 18 & 10 & $50 \%$ & $40 \%$ & $100 \%$ & $0 \%$ & 0 \\
\hline hs95 & 4 & 6 & 2 & 1 & $0 \%$ & $0 \%$ & $100 \%$ & $0 \%$ & 0 \\
\hline hs96 & 4 & 6 & 2 & 1 & $0 \%$ & $0 \%$ & $100 \%$ & $0 \%$ & 0 \\
\hline hs97 & 4 & 6 & 29 & 18 & $83 \%$ & $77 \%$ & $100 \%$ & $0 \%$ & 0 \\
\hline hs98 & 4 & 6 & 29 & 18 & $83 \%$ & $77 \%$ & $100 \%$ & $0 \%$ & 0 \\
\hline hs99 & 2 & 7 & 197 & 196 & $0 \%$ & $0 \%$ & $38 \%$ & $62 \%$ & 0 \\
\hline hs 100 & 4 & 7 & 18 & 9 & $0 \%$ & $0 \%$ & $100 \%$ & $0 \%$ & 0 \\
\hline hs100lnp & 2 & 7 & 18 & 7 & $0 \%$ & $0 \%$ & $100 \%$ & $0 \%$ & 0 \\
\hline $\mathrm{hs} 100 \mathrm{mod}$ & 4 & 7 & 19 & 12 & $91 \%$ & $0 \%$ & $100 \%$ & $0 \%$ & 0 \\
\hline hs101 & 5 & 7 & 140 & 100 & $64 \%$ & $13 \%$ & $100 \%$ & $0 \%$ & 0 \\
\hline hs102 & 5 & 7 & 72 & 57 & $82 \%$ & $10 \%$ & $100 \%$ & $0 \%$ & 0 \\
\hline hs103 & 5 & 7 & 63 & 54 & $78 \%$ & $12 \%$ & $100 \%$ & $0 \%$ & 0 \\
\hline
\end{tabular}


Table 2: Results for CUTEr test problems (continued)

\begin{tabular}{|c|c|c|c|c|c|c|c|c|c|}
\hline Problem & $m$ & $n$ & fe & itns & $\|E\|>0$ & $\|D\|>0$ & VO-itns & F-itns & M-itns \\
\hline hs 104 & 5 & 8 & 27 & 12 & $58 \%$ & $91 \%$ & $100 \%$ & $0 \%$ & 0 \\
\hline hs105 & 1 & 8 & 14 & 9 & $55 \%$ & $55 \%$ & $100 \%$ & $0 \%$ & 0 \\
\hline hs106 & 6 & 8 & $\mathrm{~F}$ & $\mathrm{~F}$ & $99 \%$ & $99 \%$ & $18 \%$ & $82 \%$ & 0 \\
\hline hs107 & 6 & 9 & 58 & 57 & $1 \%$ & $0 \%$ & $100 \%$ & $0 \%$ & 0 \\
\hline hs 108 & 13 & 9 & 22 & 11 & $90 \%$ & $81 \%$ & $100 \%$ & $0 \%$ & 0 \\
\hline hs109 & 10 & 9 & $\mathrm{~F}$ & F & $99 \%$ & $0 \%$ & $3 \%$ & $97 \%$ & 0 \\
\hline hs111 & 3 & 10 & 19 & 12 & $58 \%$ & $0 \%$ & $100 \%$ & $0 \%$ & 0 \\
\hline hs111lnp & 3 & 10 & 19 & 12 & $58 \%$ & $0 \%$ & $100 \%$ & $0 \%$ & 0 \\
\hline hs112 & 3 & 10 & 11 & 10 & $0 \%$ & $0 \%$ & $100 \%$ & $0 \%$ & 0 \\
\hline hs 113 & 8 & 10 & 12 & 7 & $0 \%$ & $0 \%$ & $100 \%$ & $0 \%$ & 0 \\
\hline hs 114 & 11 & 10 & 47 & 45 & $91 \%$ & $95 \%$ & $85 \%$ & $15 \%$ & 0 \\
\hline hs116 & 14 & 13 & $\mathrm{~F}$ & $\mathrm{~F}$ & $99 \%$ & $99 \%$ & $12 \%$ & $88 \%$ & 0 \\
\hline hs117 & 5 & 15 & 11 & 6 & $50 \%$ & $33 \%$ & $100 \%$ & $0 \%$ & 0 \\
\hline hs118 & 17 & 15 & 4 & 3 & $0 \%$ & $0 \%$ & $100 \%$ & $0 \%$ & 0 \\
\hline hs119 & 8 & 16 & 27 & 26 & $0 \%$ & $0 \%$ & $100 \%$ & $0 \%$ & 0 \\
\hline hs 268 & 5 & 5 & 10 & 4 & $0 \%$ & $0 \%$ & $100 \%$ & $0 \%$ & 0 \\
\hline $1 \mathrm{ch}$ & 1 & 300 & 54 & 29 & $96 \%$ & $0 \%$ & $83 \%$ & $17 \%$ & 0 \\
\hline lukvle1 & 98 & 100 & 17 & 10 & $40 \%$ & $0 \%$ & $100 \%$ & $0 \%$ & 0 \\
\hline lukvle3 & 2 & 100 & 15 & 8 & $0 \%$ & $0 \%$ & $100 \%$ & $0 \%$ & 0 \\
\hline lukvle6 & 49 & 99 & $\mathrm{~F}$ & $\mathrm{~F}$ & $39 \%$ & $0 \%$ & $11 \%$ & $89 \%$ & 0 \\
\hline lukvle7 & 4 & 100 & 19 & 12 & $50 \%$ & $0 \%$ & $100 \%$ & $0 \%$ & 0 \\
\hline lukvle8 & 98 & 100 & F & $F$ & $10 \%$ & $0 \%$ & $20 \%$ & $77 \%$ & 8 \\
\hline lukvle9 & 6 & 100 & 49 & 23 & $52 \%$ & $0 \%$ & $100 \%$ & $0 \%$ & 0 \\
\hline lukvle10 & 98 & 100 & 235 & 178 & $56 \%$ & $0 \%$ & $42 \%$ & $58 \%$ & 0 \\
\hline lukvle13 & 64 & 98 & 185 & 181 & $99 \%$ & $0 \%$ & $23 \%$ & $77 \%$ & 0 \\
\hline lukvle14 & 64 & 98 & F & F & $0 \%$ & $0 \%$ & $6 \%$ & $93 \%$ & 0 \\
\hline lukvle16 & 72 & 97 & 511 & 503 & $52 \%$ & $0 \%$ & $11 \%$ & $88 \%$ & 1 \\
\hline mss 1 & 73 & 90 & F & $\mathrm{F}$ & $99 \%$ & $0 \%$ & $7 \%$ & $93 \%$ & 0 \\
\hline maratos & 1 & 2 & 23 & 10 & $10 \%$ & $0 \%$ & $100 \%$ & $0 \%$ & 0 \\
\hline mwright & 3 & 5 & 13 & 6 & $0 \%$ & $0 \%$ & $100 \%$ & $0 \%$ & 0 \\
\hline orthrdm2 & 100 & 203 & 7 & 5 & $0 \%$ & $0 \%$ & $100 \%$ & $0 \%$ & 0 \\
\hline orthrds 2 & 100 & 203 & 142 & 80 & $66 \%$ & $0 \%$ & $88 \%$ & $12 \%$ & 0 \\
\hline orthrega & 64 & 133 & 37 & 21 & $61 \%$ & $0 \%$ & $100 \%$ & $0 \%$ & 0 \\
\hline orthregb & 6 & 27 & 5 & 4 & $75 \%$ & $0 \%$ & $100 \%$ & $0 \%$ & 0 \\
\hline orthregc & 10 & 25 & 8 & 7 & $0 \%$ & $0 \%$ & $100 \%$ & $0 \%$ & 0 \\
\hline orthregd & 10 & 23 & 25 & 24 & $4 \%$ & $0 \%$ & $100 \%$ & $0 \%$ & 0 \\
\hline orthrgdm & 10 & 23 & 401 & 209 & $85 \%$ & $0 \%$ & $31 \%$ & $69 \%$ & 0 \\
\hline orthrgds & 76 & 155 & 16 & 7 & $0 \%$ & $0 \%$ & $100 \%$ & $0 \%$ & 0 \\
\hline s316-322 & 1 & 2 & 10 & 7 & $0 \%$ & $0 \%$ & $100 \%$ & $0 \%$ & 0 \\
\hline
\end{tabular}

\section{Summary and Future Work}

In this paper we consider the formulation and analysis of an SQP method for solving general nonlinear optimization problems. An algorithm is proposed that combines 
the favorable properties of augmented Lagrangian methods, conventional SQP methods and stabilized SQP methods. Numerical results given in Section 6 for a simple MATLAB implementation indicate that the proposed method is robust and often exhibits fast local convergence. However, further analysis is required to determine the conditions under which the method inherits the superlinear convergence rate associated with stabilized SQP on degenerate problems.

The use of exact second-derivatives presents a significant challenge to the formulation of robust and efficient SQP methods. A key contribution of this paper is a convexification procedure that provides a convex QP subproblem based on exact second derivatives. This approach provides a first step towards an effective and efficient way of incorporating exact second derivatives in the inequality constrained QP subproblem of an SQP method.

One possible extension of the method is the use of additional regularization in the form of explicit bounds on the dual variables in the QP subproblem. For reasons of brevity, this refinement is not considered here. However, explicit temporary bounds on the dual variables are easily incorporated in the primal-dual QP subproblem (see, e.g., Robinson [46], and Gill and Robinson [23]). The formulation of improved update strategies for the regularization parameter $\mu^{R}$ is the focus of current research. It is anticipated that such strategies will allow the use of projected gradient methods for the computation of an approximate solution of each QP subproblem when far from a solution (see Friedlander and Leyffer [20]). Approaches such as this should allow future implementations of the method to solve larger problems than is possible by current SQP methods. In addition, reliable techniques that allow the rapid decrease of $\mu^{R}$ near a solution should give superlinear convergence under the standard assumptions.

\section{Acknowledgments}

We would like to thank Slava Kungurtsev for numerous discussions during the preparation of this paper.

\section{References}

[1] P. T. Boggs and J. W. Tolle. Sequential quadratic programming. In Acta Numerica, 1995, volume 4 of Acta Numer., pages 1-51. Cambridge Univ. Press, Cambridge, 1995. 2

[2] I. Bongartz, A. R. Conn, N. I. M. Gould, and P. L. Toint. CUTE: Constrained and unconstrained testing environment. ACM Trans. Math. Software, 21(1):123-160, 1995. 27

[3] R. Byrd, J. Nocedal, R. Waltz, and Y. Wu. On the use of piecewise linear models in nonlinear programming. Math. Program., pages 1-36, 2010. 10.1007/s10107-011-0492-9. 19

[4] A. R. Conn, N. Gould, A. Sartenaer, and P. L. Toint. Convergence properties of an augmented Lagrangian algorithm for optimization with a combination of general equality and linear constraints. SIAM J. Optim., 6(3):674-703, 1996. 3

[5] A. R. Conn, N. I. M. Gould, and P. L. Toint. Testing a class of method for solving minimization problems with simple bounds on the variables. Preprint, 1986. 9

[6] A. R. Conn, N. I. M. Gould, and P. L. Toint. Global convergence of a class of trust region algorithms for optimization with simple bounds. SIAM J. Numer. Anal., 25:433-460, 1988. 3 
[7] A. R. Conn, N. I. M. Gould, and P. L. Toint. A comprehensive description of LANCELOT. Technical Report 91/10, Département de Mathématique, Facultés Universitaires de Namur, 1991. 15

[8] A. R. Conn, N. I. M. Gould, and P. L. Toint. A globally convergent augmented Lagrangian algorithm for optimization with general constraints and simple bounds. SIAM J. Numer. Anal., 28:545-572, 1991. 3, 15

[9] A. R. Conn, N. I. M. Gould, and P. L. Toint. LANCELOT: a Fortran package for large-scale nonlinear optimization (Release A). Lecture Notes in Computation Mathematics 17. Springer Verlag, Berlin, Heidelberg, New York, London, Paris and Tokyo, 1992. 3

[10] A. R. Conn, N. I. M. Gould, and P. L. Toint. Large-scale nonlinear constrained optimization. Technical Report 92/2, Département de Mathématique, Facultés Universitaires de Namur, 1992. 3

[11] L. B. Contesse. Une caractérisation complète des minima locaux en programmation quadratique. Numer. Math., 34:315-332, 1980. 2

[12] F. E. Curtis and J. Nocedal. Flexible penalty functions for nonlinear constrained optimization. IMA J. Numer. Anal., 28(4):749-769, 2008. 12

[13] D. Fernández and M. Solodov. Local convergence of exact and inexact augmented Lagrangian methods under the second-order sufficiency condition, 2010. IMPA preprint A677. 18

[14] D. Fernández and M. Solodov. Stabilized sequential quadratic programming for optimization and a stabilized Newton-type method for variational problems. Math. Program., 125(1, Ser. A):47-73, 2010. 2, 6, 18

[15] R. Fletcher. An $\ell_{1}$ penalty method for nonlinear constraints. In P. T. Boggs, R. H. Byrd, and R. B. Schnabel, editors, Numerical Optimization 1984, pages 26-40, Philadelphia, 1985. 2

[16] R. Fletcher and S. Leyffer. User manual for filterSQP. Technical Report NA/181, Dept. of Mathematics, University of Dundee, Scotland, 1998. 2

[17] A. Forsgren. Inertia-controlling factorizations for optimization algorithms. Appl. Num. Math., 43:91-107, 2002. 20, 28

[18] A. Forsgren and P. E. Gill. Primal-dual interior methods for nonconvex nonlinear programming. SIAM J. Optim., 8:1132-1152, 1998. 6, 20, 28

[19] A. Forsgren, P. E. Gill, and W. Murray. On the identification of local minimizers in inertiacontrolling methods for quadratic programming. SIAM J. Matrix Anal. Appl., 12:730-746, 1991. 2

[20] M. P. Friedlander and S. Leyffer. Global and finite termination of a two-phase augmented Lagrangian filter method for general quadratic programs. SIAM J. Sci. Comput., 30(4):17061729, 2008. 34

[21] P. E. Gill, W. Murray, and M. A. Saunders. SNOPT: An SQP algorithm for large-scale constrained optimization. SIAM Rev., 47:99-131, 2005. 2, 9, 19, 29

[22] P. E. Gill, W. Murray, M. A. Saunders, and M. H. Wright. Some theoretical properties of an augmented Lagrangian merit function. In P. M. Pardalos, editor, Advances in Optimization and Parallel Computing, pages 101-128. North Holland, North Holland, 1992. 2

[23] P. E. Gill and D. P. Robinson. A primal-dual augmented Lagrangian. Computational Optimization and Applications, pages 1-25, 2010. 3, 6, 7, 21, 27, 34

[24] P. E. Gill and E. Wong. Sequential quadratic programming methods. In J. Lee and S. Leyffer, editors, Mixed Integer Nonlinear Programming, volume 154 of The IMA Volumes in Mathematics and its Applications, pages 147-224. Springer New York, 2012. 10.1007/978-1-46141927-3_6. 2, 3

[25] P. E. Gill and E. Wong. Methods for convex and general quadratic programming. Center for Computational Mathematics Report 13-1, University of California, San Diego, La Jolla, CA, 2013. 20 
[26] P. E. Gill and E. Wong. A regularized method for convex and general quadratic programming. Center for Computational Mathematics Report 13-3, Department of Mathematics, University of California, San Diego, La Jolla, CA, 2013. 28

[27] N. I. M. Gould. On modified factorizations for large-scale linearly-constrained optimization. SIAM J. Optim., 9:1041-1063, 1999. 20

[28] N. I. M. Gould, D. Orban, and P. L. Toint. CUTEr and SifDec: A constrained and unconstrained testing environment, revisited. ACM Trans. Math. Software, 29(4):373-394, 2003. 3, 27

[29] N. I. M. Gould and D. P. Robinson. A second derivative SQP method: Global convergence. SIAM J. Optim., 20(4):2023-2048, 2010. 2, 19

[30] N. I. M. Gould and D. P. Robinson. A second derivative SQP method: Local convergence and practical issues. SIAM J. Optim., 20(4):2049-2079, 2010. 2

[31] W. W. Hager. Stabilized sequential quadratic programming. Comput. Optim. Appl., 12(13):253-273, 1999. Computational optimization - a tribute to Olvi Mangasarian, Part I. 2, 6

[32] S. P. Han. Superlinearly convergent variable metric algorithms for general nonlinear programming problems. Math. Programming, 11(3):263-282, 1976/77. 2

[33] M. R. Hestenes. Multiplier and gradient methods. J. Optim. Theory Appl., 4:303-320, 1969. 3

[34] W. Hock and K. Schittkowski. Test Examples for Nonlinear Programming Codes. Lecture Notes in Economics and Mathematical Systems 187. Springer Verlag, Berlin, Heidelberg and New York, 1981. 27

[35] A. F. Izmailov and M. V. Solodov. On attraction of linearly constrained Lagrangian methods and of stabilized and quasi-Newton SQP methods to critical multipliers. Math. Program., 126(2, Ser. A):231-257, 2011. 2, 18

[36] A. F. Izmailov and M. V. Solodov. Stabilized SQP revisited. Math. Program., 133:93-120, 2012. 18

[37] V. Kungurtsev. Second-Derivative Sequential Quadratic Programming Methods for Nonlinear Optimization. PhD thesis, Department of Mathematics, University of California, San Diego, February 2013. 28, 29

[38] D.-H. Li and L. Qi. A stabilized SQP method via linear equations. Technical Report AMR00/5, School of Mathematics, University of New South Wales, Sydney, 2000. 6

[39] C. M. Maes. A Regularized Active-Set Method for Sparse Convex Quadratic Programming. $\mathrm{PhD}$ thesis, Institute for Computational and Mathematical Engineering, Stanford University, Stanford, CA, August 2010. 3

[40] N. Maratos. Exact Penalty Function Algorithms for Finite-Dimensional and Control Optimization Problems. PhD thesis, Department of Computing and Control, University of London, 1978. 11

[41] J. Morales, J. Nocedal, and Y. Wu. A sequential quadratic programming algorithm with an additional equality constrained phase. IMA Journal of Numerical Analysis, DOI: 10.1093/imanum/drq037, 2011. 2

[42] J. Nocedal and S. J. Wright. Numerical Optimization. Springer-Verlag, New York, 1999. 18, 29

[43] C. Oberlin and S. J. Wright. Active set identification in nonlinear programming. SIAM J. Optim., 17(2):577-605, 2006. 2

[44] M. J. D. Powell. A method for nonlinear constraints in minimization problems. In R. Fletcher, editor, Optimization, pages 283-298, London and New York, 1969. Academic Press. 3, 6

[45] M. J. D. Powell. A fast algorithm for nonlinearly constrained optimization calculations. Technical Report 77/NA 2, Department of Applied Mathematics and Theoretical Physics, University of Cambridge, England, 1977. 2 
[46] D. P. Robinson. Primal-Dual Methods for Nonlinear Optimization. PhD thesis, Department of Mathematics, University of California, San Diego, September 2007. 6, 7, 15, 19, 34

[47] K. Schittkowski. The nonlinear programming method of Wilson, Han, and Powell with an augmented Lagrangian type line search function. I. Convergence analysis. Numer. Math., 38(1):83-114, 1981/82. 2

[48] K. Schittkowski. The nonlinear programming method of Wilson, Han, and Powell with an augmented Lagrangian type line search function. II. An efficient implementation with linear least squares subproblems. Numer. Math., 38(1):115-127, 1981/82. 2

[49] A. Wächter and L. T. Biegler. Line search filter methods for nonlinear programming: motivation and global convergence. SIAM J. Optim., 16(1):1-31 (electronic), 2005. 20

[50] S. J. Wright. Superlinear convergence of a stabilized SQP method to a degenerate solution. Comput. Optim. Appl., 11(3):253-275, 1998. 2, 6

[51] S. J. Wright. Modifying SQP for degenerate problems. SIAM J. Optim., 13(2):470-497, 2002. 2 